\title{
Critical analysis of the potential for microRNA biomarkers in breast cancer management
}

This article was published in the following Dove Press journal:

Breast Cancer:Targets and Therapy

23 February 2015

Number of times this article has been viewed

\section{Carrie R Graveel' Heather M Calderone ${ }^{2}$ Jennifer J Westerhuis ${ }^{2}$ Mary E Winn ${ }^{3}$ Lorenzo F Sempere ${ }^{2}$}

'Breast Cancer Signaling and Therapeutics Team, Program in Molecular Oncology and Pre-clinical Therapeutics, Center for Cancer and Cell Biology, ${ }^{2}$ Laboratory of microRNA Diagnostics and Therapeutics, Program in Skeletal Disease and Tumor Microenvironment. Center for Cancer and Cell Biology, ${ }^{3}$ Bioinformatics and Biostatistics Core, Program for Technologies and Cores, Van Andel Research Institute, Grand Rapids, MI, USA

\section{Video abstract}

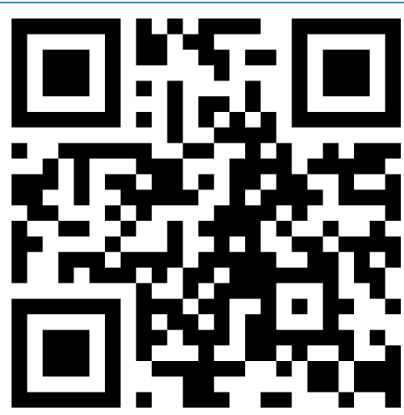

Point your SmartPhone at the code above. If you have a QR code reader the video abstract will appear. Or use: http://dvpr.es//CNPZtII

Correspondence: Lorenzo F Sempere Laboratory of microRNA Diagnostics and Therapeutics, Program in Skeletal Disease and Tumor Microenvironment, Center for Cancer and Cell Biology, Van Andel Research Institute, 333 Bostwick Ave NE, Grand Rapids, MI 49503, USA

Tel + I 6162345530

Email lorenzo.sempere@vai.org
Abstract: Breast cancer is a complex and heterogeneous disease. Signaling by estrogen receptor (ER), progesterone receptor (PR), and/or human EGF-like receptor 2 (HER2) is a main driver in the development and progression of a large majority of breast tumors. Molecular characterization of primary tumors has identified major subtypes that correlate with ER/PR/HER2 status, and also subgroup divisions that indicate other molecular and cellular features of the tumors. While some of these research findings have been incorporated into clinical practice, several challenges remain to improve breast cancer management and patient survival, for which the integration of novel biomarkers into current practice should be beneficial. microRNAs (miRNAs) are a class of short non-coding regulatory RNAs with an etiological contribution to breast carcinogenesis. miRNA-based diagnostic and therapeutic applications are rapidly emerging as novel potential approaches to manage and treat breast cancer. Rapid technological development enables specific and sensitive detection of individual miRNAs or the entire miRNome in tissues, blood, and other biological specimens from breast cancer patients. This review focuses on recent miRNA research and its potential to address unmet clinical needs and challenges. The four sections presented discuss miRNA findings in the context of the following clinical challenges: biomarkers for early detection; prognostic and predictive biomarkers for treatment decisions using targeted therapies against ER and HER2; diagnostic and prognostic biomarkers for subgrouping of triple-negative breast cancer, for which there are currently no targeted therapies; and biomarkers for monitoring and characterization of metastatic breast cancer. The review concludes with a critical analysis of the current state of miRNA breast cancer research and the need for further studies using large patient cohorts under well-controlled conditions before considering the clinical implementation of miRNA biomarkers.

Keywords: miRNA, miR, blood, formalin-fixed paraffin-embedded tissues, diagnostics FFPE tissues

\section{Introduction}

Breast cancer is a highly heterogeneous disease that has multiple subtypes with distinct clinical outcomes. Clinically, breast cancers are classified by hormone receptor status, including estrogen receptor (ER), progesterone receptor (PR), and human EGF-like receptor 2 (HER2) receptor expression, as well as by tumor grade. In the last decade, gene expression analyses have given us a more thorough understanding of the molecular heterogeneity of breast cancer. Breast cancer is currently classified into six molecular intrinsic subtypes: luminal A, luminal B, HER2 ${ }^{+}$, normal-like, basal, and claudin-low. ${ }^{1,2}$ Luminal cancers are commonly dependent on hormone (ER and/or PR) signaling and have the best outcome. Basal and claudin-low cancers significantly overlap with the immunohistological subtype referred to as triple-negative breast cancer (TNBC), which 
lacks ER, PR, and HER2 expression. Basal/TNBC cancers have the worst outcome and there are currently no approved targeted therapies for these patients. ${ }^{3,4}$

Breast cancer is a forerunner in the use of targeted therapeutic approaches. Endocrine therapy is standard treatment for $\mathrm{ER}^{+}$breast cancers. The development of trastuzumab $\left(\right.$ Herceptin $\left.^{\circledR}\right)$ treatment for HER2 ${ }^{+}$breast cancers provides clear evidence for the value in combining prognostic biomarkers with targeted therapies. Even though early detection and targeted therapies have significantly lowered breast cancer-related mortality rates, there are still hurdles that need to be overcome. The most significant of these are: 1) improved detection of neoplastic lesions and identification of high-risk individuals (Tables 1 and 2);2) the development of predictive biomarkers for carcinomas that will develop resistance to hormone therapy (Table 3 ) or trastuzumab treatment (Table 4); 3) the development of clinical biomarkers to distinguish TNBC subtypes (Table 5); and 4) the lack of effective monitoring methods and treatments for metastatic breast cancer (MBC; Table 6). In order to make advances in these areas, we must understand the heterogeneous landscape of individual tumors, develop predictive and prognostic biomarkers that can be affordably used at the clinical level, and identify unique therapeutic targets. In this review, we discuss recent findings on microRNAs (miRNAs) research aimed at addressing these challenges.

Numerous in vitro and in vivo models have demonstrated that dysregulation of individual miRNAs influences signaling networks involved in breast cancer progression. These studies suggest potential applications for miRNAs as both disease biomarkers and therapeutic targets for clinical intervention. Here, we provide a brief overview of miRNA biogenesis and detection methods with implications for breast cancer management. We also discuss the potential clinical applications for miRNAs in early disease detection, for prognostic indications and treatment selection, as well as diagnostic opportunities in TNBC and metastatic disease.

\section{miRNA biogenesis and regulatory mechanisms of gene control}

miRNAs are short non-coding regulatory RNAs that generally regulate gene expression at the post-transcriptional level. ${ }^{5}$ The main molecular mechanism for this regulatory mode consists of mature miRNA (18-24 nt) binding to partially complementary sites on the $3^{\prime}$-UTR (untranslated region) of target mRNAs. ${ }^{5,6}$ The mature miRNA is associated with the Argonaute-containing multi-protein RNA-induced silencing complex (miRISC). miRNA interaction with a target RNA brings the miRISC into close proximity to the mRNA, causing mRNA degradation and/or translational repression. Due to the low specificity of binding, a single miRNA can interact with hundreds of mRNAs and coordinately modulate expression of the corresponding proteins. The extent of miRNA-mediated regulation of different target genes varies and is influenced by the context and cell type expressing the miRNA. ${ }^{5}$

\section{Methods for miRNA detection in blood and tissues}

Most miRNAs are transcribed by RNA polymerase II as part of a host gene transcript or as individual or polycistronic miRNA transcripts. ${ }^{5,7}$ As such, miRNA expression can be regulated at epigenetic and transcriptional levels. ${ }^{8,9} 5^{\prime}$ capped and polyadenylated primary miRNA transcripts are shortlived in the nucleus where the microprocessor multi-protein complex recognizes and cleaves the miRNA precursor hairpin (pre-miRNA; about $70 \mathrm{nt}$ ). ${ }^{5,10}$ pre-miRNA is exported out of the nucleus via the XPO5 pathway., ${ }^{5,10}$ In the cytoplasm, the RNase type III Dicer cleaves mature miRNA (19-24 nt) from pre-miRNA. In most cases, one of the pre-miRNA arms is preferentially processed and stabilized as mature miRNA (miR-\#), while the other arm is not as efficiently processed or is quickly degraded (miR-\#*). In some cases, both arms can be processed at similar rates and accumulate in similar amounts. The initial nomenclature captured these differences in mature miRNA levels as ' $\mathrm{miR}-\# / \mathrm{miR}-\# *$, and 'miR-\#-5p/miR-\#-3p', respectively. More recently, the nomenclature has been unified to ' $\mathrm{miR}-\#-5 \mathrm{p} / \mathrm{miR}-\#-3 \mathrm{p}$ ' and simply reflects the hairpin location from which each RNA arm is processed, since they may each produce functional miRNAs that associate with RISC ${ }^{11}$ (note that in this review we present miRNA names as originally published, so those names may not adhere to the newer guidelines). Molecular aberrations that interfere with miRNA processing, export, and/or maturation affect mature miRNA levels and biological activity. Accordingly, most miRNA detection methods focus on the analysis of mature miRNA as it most closely correlates with miRNA activity, is more long-lived, and more resistant to nuclease degradation than a primary miRNA transcript, a pre-miRNA hairpin, or mRNAs.

While the short length of mature miRNA presents advantages as a robust bioanalyte, it also presents challenges for specific and sensitive detection. Capture-probe microarray and bead platforms were major breakthroughs that have enabled high-throughput characterization of miRNA expression in 

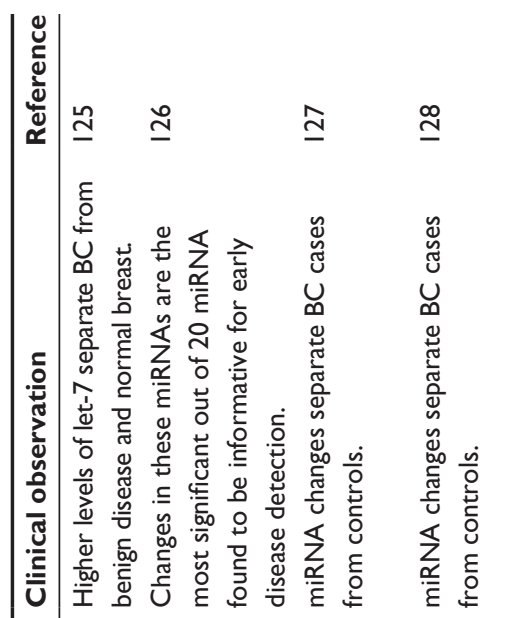

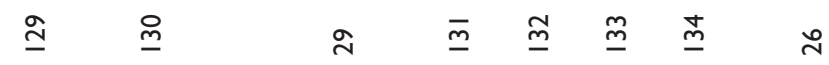

๙
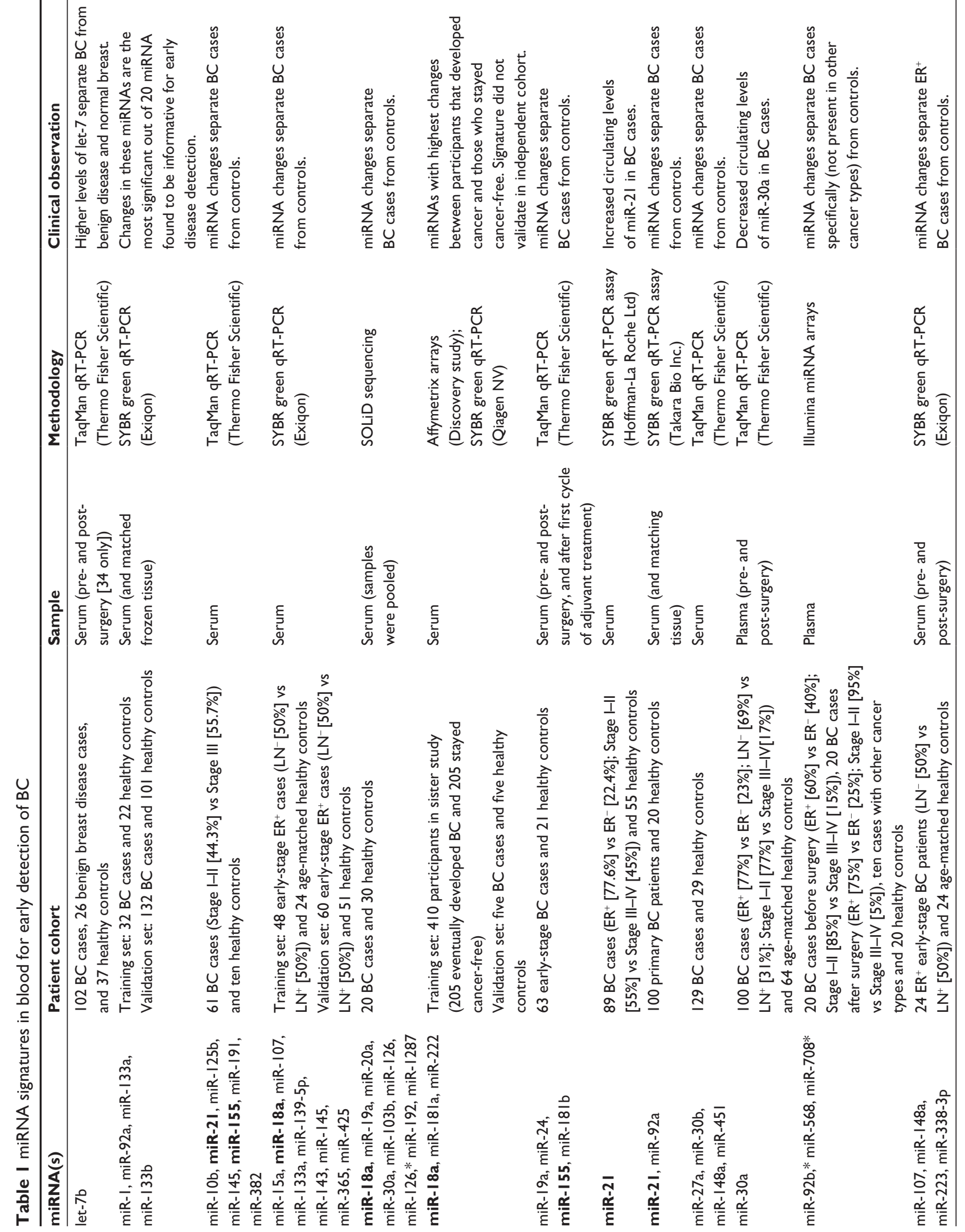

量高
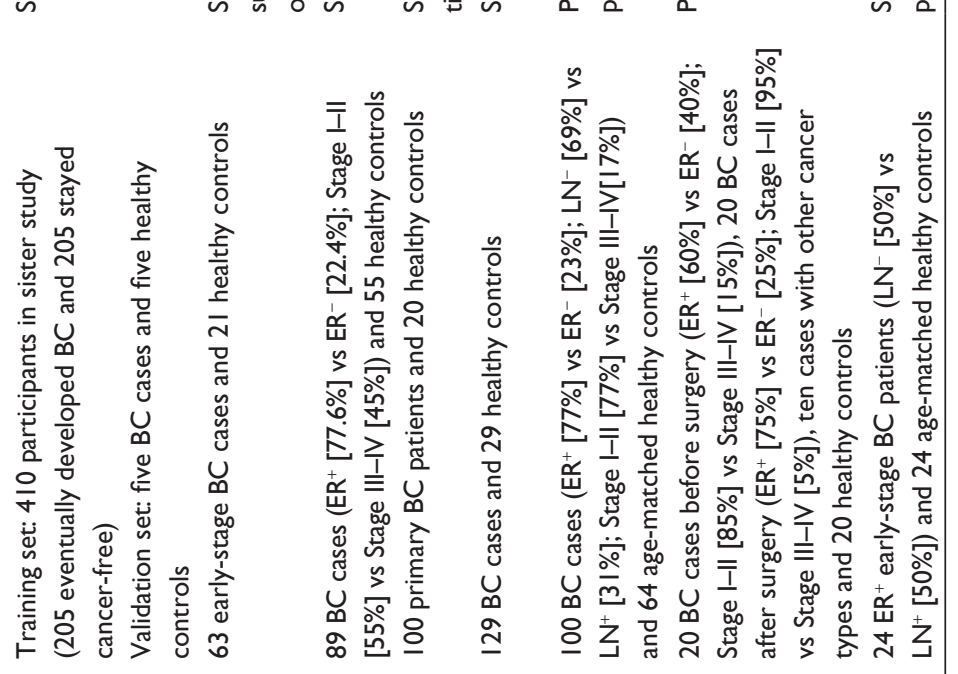

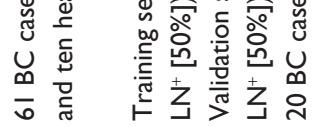

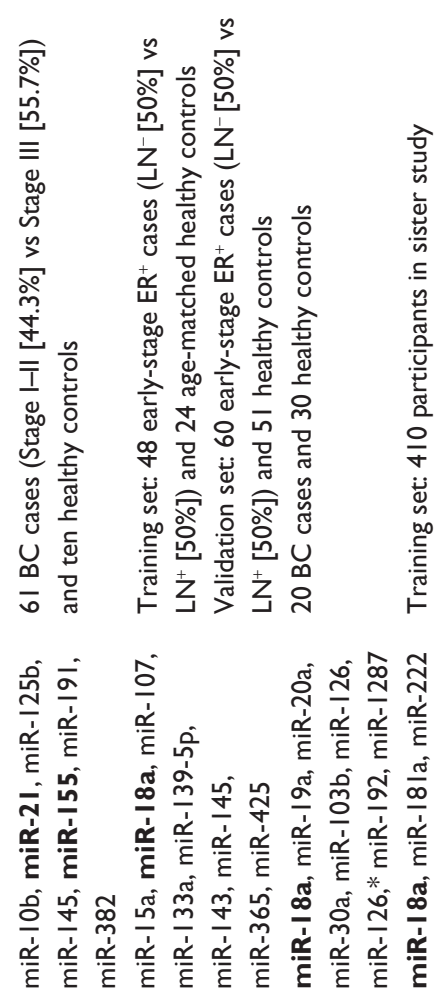

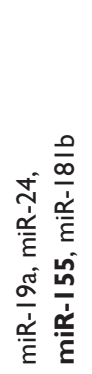

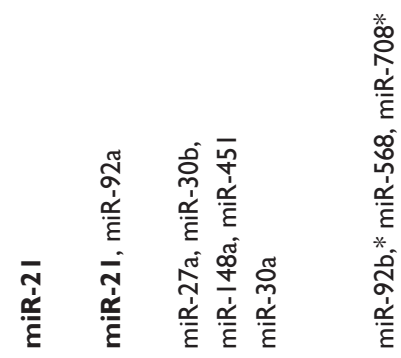

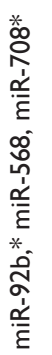

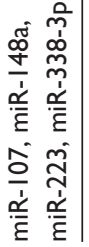




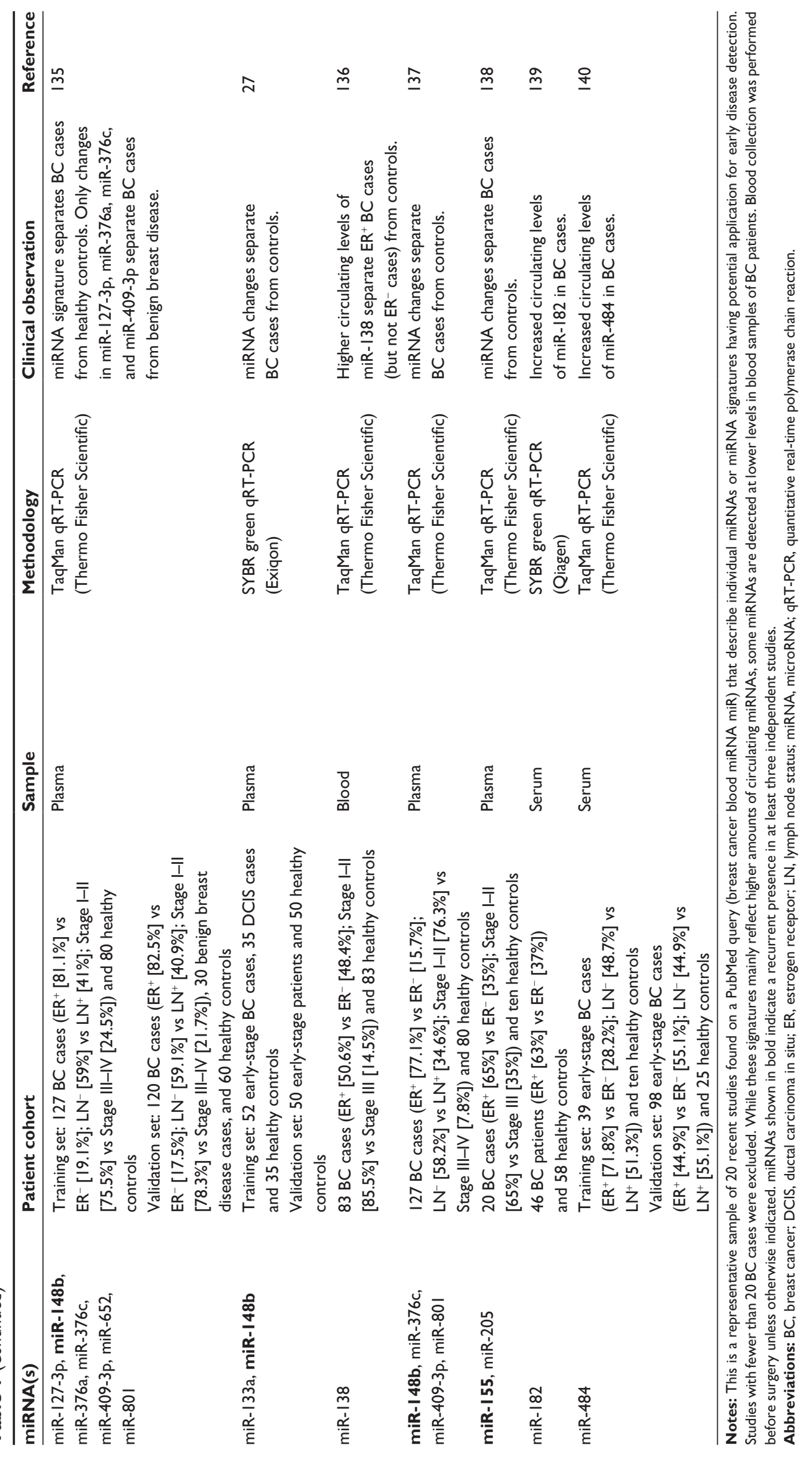


Table 2 miRNA-related risk loci associated with BC

\begin{tabular}{|c|c|c|c|c|c|}
\hline Gene locus & SNP & Comments & Population & Clinical observation & Reference \\
\hline \multirow[t]{10}{*}{ MIR27A } & rs895919 *C & & Asians & No risk association & 141 \\
\hline & & & Caucasians & Protective & \\
\hline & & & Jewish BRCA2 carriers & Increased risk & 142 \\
\hline & rs895819 A/G & pre-miRNA & Caucasian & Decreased risk & 143 \\
\hline & & pre-miRNA & Asians & No risk association & 144 \\
\hline & & & Caucasians & Decreased risk & \\
\hline & & pre-miRNA & Chinese (young) & Decreased risk & 35 \\
\hline & & pre-miRNA & Chinese & Decreased risk & 34 \\
\hline & & & Asians & No risk association & 31 \\
\hline & & & Caucasians & No risk association & \\
\hline MIR34B cluster & rs4938723 T/C & & African Americans & Increased survival & 145 \\
\hline \multirow[t]{2}{*}{ MIRIO0 } & rs 1834306 G/A & & African Americans & No risk association & 33 \\
\hline & & & European Americans & Decreased overall risk & \\
\hline \multirow[t]{2}{*}{ MIRIOI-2 } & $\mathrm{rs} 1053872 \mathrm{C} / \mathrm{G}$ & Primary transcript & Chinese & Increased risk & 38 \\
\hline & $\mathrm{rs} 462480 \mathrm{~A} / \mathrm{C}$ & Primary transcript & Chinese & Increased risk & 38 \\
\hline \multirow[t]{2}{*}{ MIRI06B } & $\mathrm{rs} / 527423 \mathrm{~A} / \mathrm{G}$ & & African Americans & No risk association & 33 \\
\hline & & & European Americans & Increased overall risk & \\
\hline \multirow[t]{2}{*}{$M I R / 22 A$} & rsI7669 A/G & & African Americans & Decreased risk of $E R^{+} B C$ & 33 \\
\hline & & & European Americans & No risk association & \\
\hline \multirow[t]{9}{*}{$M I R / 46 A$} & rs2910164 G/C & & Italian & Earlier age of onset & 146 \\
\hline & & & Caucasians & No risk association & 147 \\
\hline & & & Chinese & No risk association & 83 \\
\hline & & & Asians & No risk association & 38 \\
\hline & & & Caucasians & No risk association & \\
\hline & & & Asians & Decreased risk (C allele) & 144 \\
\hline & & & Asians & No risk association & 31 \\
\hline & & & Caucasians & No risk association & \\
\hline & & & Chinese & No risk association & 36 \\
\hline \multirow[t]{5}{*}{ MIRI 49} & rs2292832 T/G & & Asians & No risk association & 38 \\
\hline & & & Caucasians & No risk association & \\
\hline & & & Chinese & No risk association & 36 \\
\hline & & & Asians & No risk association & 31 \\
\hline & & & Caucasians & No risk association & \\
\hline \multirow[t]{2}{*}{ MIRI85 } & rs200859I C/T & & African Americans & Reduced risk & 145 \\
\hline & rs887205 A/G & & African Americans & Reduced risk & 145 \\
\hline \multirow[t]{13}{*}{ MIRI96A-2 } & $\mathrm{rs} 11614913 \mathrm{~T} / \mathrm{C}$ & & Korean & Survival of HER2 ${ }^{+}$cases & 148 \\
\hline & & & Italian and German & No risk association & 37 \\
\hline & & & Asians & Decreased risk & $|4|$ \\
\hline & & & Caucasians & No risk association & \\
\hline & & & Brazilian & Decreased risk & 149 \\
\hline & & & Caucasian & Decreased risk & 147 \\
\hline & & & Chinese and Korean & Decreased risk & 32 \\
\hline & & & Chinese & Increased risk & 36 \\
\hline & & & Chinese & Increased risk & 83 \\
\hline & & & African Americans & No risk association & 33 \\
\hline & & & European Americans & No risk association & \\
\hline & & & Asians & No risk association & 31 \\
\hline & & & Caucasians & No risk association & \\
\hline \multirow[t]{2}{*}{ MIR204 } & rs786I254 G & & African Americans & Decreased risk of $E R^{-} B C$ & 33 \\
\hline & & & European Americans & No risk association & \\
\hline MIR206 & rs6920648 A/G & & African Americans & Increased survival & 145 \\
\hline \multirow[t]{2}{*}{ MIR219 } & rs 107822 G/A & & African Americans & Increased risk of $E R^{-} B C$ & 33 \\
\hline & & & European Americans & No risk association & \\
\hline \multirow[t]{2}{*}{ MIR33I } & rs III 07973 A/G & & African Americans & No risk association & 33 \\
\hline & & & European Americans & Increased overall risk & \\
\hline \multirow[t]{2}{*}{ MIR373 } & rs I $2983273 \mathrm{~T} / \mathrm{C}$ & & Asians & No risk association & 38 \\
\hline & & & Caucasians & No risk association & \\
\hline
\end{tabular}


Table 2 (Continued)

\begin{tabular}{|c|c|c|c|c|c|}
\hline Gene locus & SNP & Comments & Population & Clinical observation & Reference \\
\hline \multirow[t]{4}{*}{ MIR423 } & rs6505I62 A/C & pre-miRNA & Caucasian & Reduced risk & 150 \\
\hline & & & Jewish BRCA2 carriers & Later age of onset & 142 \\
\hline & & & Asians & No risk association & 38 \\
\hline & & & Caucasians & No risk association & \\
\hline \multirow[t]{2}{*}{ MIR455 } & rs2060I33 C/G & & African Americans & No risk association & 33 \\
\hline & & & European Americans & Decreased risk of $E R^{+} B C$ & \\
\hline \multirow[t]{2}{*}{ MIR487 } & rs $1951032 \mathrm{G} / \mathrm{A}$ & & African Americans & No risk association & 33 \\
\hline & & & European Americans & Increased overall risk & \\
\hline \multirow[t]{2}{*}{ MIR495 } & rs228I6II C/A & & African Americans & No risk association & 33 \\
\hline & & & European Americans & Increased risk of $\mathrm{ER}^{-} \mathrm{BC}$ & \\
\hline \multirow[t]{13}{*}{ MIR499 } & rs3746444 A/G & & Chinese & Increased risk & 36 \\
\hline & & & Asians & Increased risk & $15 \mid$ \\
\hline & & & Caucasians & No risk association & \\
\hline & & & Asians & Increased risk & 152 \\
\hline & & & Caucasians & No risk association & \\
\hline & & & Chinese & Increased risk & 32 \\
\hline & & & Caucasians & No risk association & 147 \\
\hline & rs3746444 T/C & & Asians & Increased risk & 153 \\
\hline & & & Caucasians & No risk association & \\
\hline & & & Asians & Increased risk & 31 \\
\hline & & & Caucasians & No risk association & \\
\hline & & & Asians & Decreased risk & $|4|$ \\
\hline & & & Caucasians & No risk association & \\
\hline \multirow[t]{2}{*}{ MIR5 I 3A-2 } & rs20I8562 A/G & & African Americans & Increased overall risk & 33 \\
\hline & & & European Americans & No risk association & \\
\hline \multirow[t]{2}{*}{ MIR544 } & $\mathrm{rs} 10144 \mid 93 \mathrm{~A} / \mathrm{T}$ & & African Americans & No risk association & 33 \\
\hline & & & European Americans & Increased overall risk & \\
\hline \multirow[t]{2}{*}{ MIR548A-2 } & rs878I75 A/G & & African Americans & No risk association & 33 \\
\hline & & & European Americans & Decreased risk of $E R^{+} B C$ & \\
\hline \multirow[t]{2}{*}{ MIR595 } & rs4909238 A/G & & African Americans & Increased risk of $\mathrm{ER}^{-} \mathrm{BC}$ & 33 \\
\hline & & & European Americans & No risk association & \\
\hline \multirow[t]{2}{*}{ MIR605 } & rs $2043556 * A$ & & Asians & Decreased risk & $|4|$ \\
\hline & & & Caucasians & No risk association & \\
\hline \multirow[t]{2}{*}{ MIR606 } & rs $1226698 \mid \mathrm{G} / \mathrm{A}$ & & African Americans & Increased risk of $\mathrm{ER}^{+}$ & 33 \\
\hline & & & European Americans & No risk association & \\
\hline \multirow[t]{2}{*}{ MIR608 } & $\mathrm{rs} 4919510 \mathrm{C} / \mathrm{G}$ & & African Americans & No risk association & 33 \\
\hline & & & European Americans & Decreased risk of $E R^{-} B C$ & \\
\hline \multirow[t]{4}{*}{ MIR628 } & rs804I885 A/G & & African Americans & Decreased risk of $E R^{+} B C$ & 33 \\
\hline & & & European Americans & No risk association & \\
\hline & rs804I $044 \mathrm{C} / \mathrm{A}$ & & African Americans & Decreased risk of $\mathrm{ER}^{+}$ & 33 \\
\hline & & & European Americans & No risk association & \\
\hline \multirow[t]{2}{*}{ MIR659 } & rs5750504 T/A & & African Americans & No risk association & 33 \\
\hline & & & European Americans & Increased overall risk & \\
\hline \multirow[t]{4}{*}{ MIR758 } & rs $12586258 \mathrm{G} / \mathrm{A}$ & & African Americans & Decreased overall risk & 33 \\
\hline & & & European Americans & No risk association & \\
\hline & rs7/4I987 A/G & & African Americans & No risk association & 33 \\
\hline & & & European Americans & Increased risk of $\mathrm{ER}^{-} \mathrm{BC}$ & \\
\hline ATFI $3^{\prime}$ UTR & $\mathrm{rs} I \mathrm{II} 6957 \mathrm{I} \mathrm{C} / \mathrm{T}$ & miR-320 MRE & Jewish BRCA2 carriers & Increased risk & 142 \\
\hline BMPRIB $3^{\prime}$ UTR & $\mathrm{rs} \mid 434536 \mathrm{C} / \mathrm{T}$ & miR-I25b MRE & Caucasian & Increased risk & 43 \\
\hline BRCAI 3' UTR & rs799917 C/T & miR-638 MRE & Italian & Increased risk & 154 \\
\hline HPGD 3' UTR & rs8752 G/A & miR-485-5p MRE & Chinese & Increased risk in $\mathrm{ER}^{+}$ & 155 \\
\hline IGF-IR 3' UTR & $\mathrm{rs} 28674628 \mathrm{~A} / \mathrm{G}$ & miR-5I5-5p MRE & Jewish BRCAI carriers & Earlier age of onset & 156 \\
\hline \multirow[t]{3}{*}{ IL23R 3' UTR } & rs $10889677 \mathrm{~A} / \mathrm{C}$ & let-7 MRE & Chinese & Increased risk (A allele) & 157 \\
\hline & & & & Earlier age of onset ( $\mathrm{C}$ allele) & \\
\hline & & & & Decreased risk & 158 \\
\hline IQGAPI 3' UTR & rsI042538 A/T & miR-I24 MRE & Chinese & Decreased risk & 159 \\
\hline \multirow[t]{2}{*}{ ITGB4 3' UTR } & rs743554 A/G & miR-34a MRE predicted & Swedish & Increased risk & 160 \\
\hline & & & & Reduced survival & \\
\hline
\end{tabular}


Table 2 (Continued)

\begin{tabular}{|c|c|c|c|c|c|}
\hline miRNA locus & SNP & Comments & Population & Clinical observation & Reference \\
\hline \multirow[t]{2}{*}{ KRAS 3' UTR } & $\mathrm{rs} 61764370 \mathrm{~T} / \mathrm{G}$ & let-7 MRE & Caucasian & $\begin{array}{l}\text { Increased frequency in BRCAI } \\
\text { carriers/no risk association }\end{array}$ & 40 \\
\hline & & & & Increased risk of TNBC & 39 \\
\hline RAD52 3' UTR & rs796355I A/C & let-7 MRE & Chinese & Decreased risk & 42 \\
\hline RYR3 3' UTR & $\mathrm{rs} 1044129 \mathrm{~A} / \mathrm{G}$ & miR-367 MRE & Chinese & Increased risk and poor survival & 161 \\
\hline SET8 3' UTR & $\mathrm{rs} 16917496 \mathrm{C} / \mathrm{T}$ & miR-502 MRE & Asian & Earlier age of onset & 162 \\
\hline TGFBRI 3' UTR & rs334348 A/G & miR-628-5p MRE & Italian & Increased risk & 154 \\
\hline TGFBI Exonic & rsl982073 C/T & miR-187 MRE & Italian & Increased risk & 154 \\
\hline$X R C C I$ Exonic & rsl799782 T/C & miR-I38 MRE & Italian & Decreased risk & 154 \\
\hline \multirow[t]{6}{*}{ AGO4 } & rs735493I C/A & miRNA RISC-loading, & African Americans & Decreased overall risk & 33 \\
\hline & & miRNA-RISC activity & European Americans & No risk association & \\
\hline & $\mathrm{rs} 16822342 \mathrm{~A} / \mathrm{G}$ & & African Americans & Decreased risk of $E R^{+} B C$ & 33 \\
\hline & & & European Americans & No risk association & \\
\hline & rs3820276 G/C & & African Americans & Increased risk of $\mathrm{ER}^{+} \mathrm{BC}$ & 33 \\
\hline & & & European Americans & No risk association & \\
\hline \multirow[t]{3}{*}{ DGCR8 } & rs4I7309 G/A & Pre-miRNA & Chinese & Increased risk & 42 \\
\hline & rs960624I A/G & processing & African Americans & No risk association & 33 \\
\hline & & & European Americans & Increased risk of $\mathrm{ER}^{+} \mathrm{BC}$ & \\
\hline \multirow[t]{2}{*}{ PACT } & rs205969I G/A & miRNA-RISC activity & African Americans & No risk association & 33 \\
\hline & & & European Americans & Increased overall risk & \\
\hline \multirow[t]{2}{*}{ XPO5 } & rsII077 A/C & Pre-miRNA & African Americans & Decreased risk of $E R^{+} B C$ & 33 \\
\hline & & nuclear export & European Americans & No risk association & \\
\hline
\end{tabular}

Abbreviations: BC, breast cancer; ER, estrogen receptor; HER2, human EGF-like receptor 2; miRNA, microRNA; MRE, microRNA recognition element (ie, binding site); RISC, RNA-induced silencing complex; UTR, untranslated region.

cancer tissues. Typically, these platforms require a large amount of sample, making direct studies of blood or other biological fluids having low miRNA content difficult. Stem-loop primer reverse transcription polymerase chain reaction (RT-PCR) analysis provides an alternative platform that can detect a much lower number of miRNA copies. Such analysis was initially used as an independent validation tool for array-based expression profiling findings and is the current gold standard practice for technical validation of altered miRNA expression. High-throughput RT-PCR multiplexing platforms have enabled characterization of miRNA expression in blood. More recently, NanoString and RNA-Seq analyses have added new high-throughput tools with single molecule detection capabilities. All of these detection methods, each with unique advantages and limitations, have been applied to expression profiling of miRNAs in breast cancer tissues and blood samples from breast cancer patients. ${ }^{12-15}$

\section{miRNA biomarkers for early disease detection}

The prognosis for breast cancer patients is strongly influenced by the stage of the disease. For instance, the 5-year survival rate is $99 \%$ for localized disease, $84 \%$ for regional disease, and $24 \%$ for distant-stage disease. ${ }^{16}$ Larger tumor size also correlates with poorer prognosis. Therefore, it is essential that breast cancer lesions are diagnosed at the earliest stages. Mammography, ultrasound, magnetic resonance, and nuclear medicine are used to identify breast lesions at their earliest stages. ${ }^{17}$ Mammography is the current gold standard for breast cancer detection for women over the age of 39 years. However, its limitations include high false-positive rates $(12.1 \%-65.8 \%)^{18}$ that lead to additional imaging and biopsies, ${ }^{19}$ and low success rates in the detection of neoplastic tissue within dense breast tissue. A combination of mammography with magnetic resonance or other imaging platforms can enhance tumor detection, but this additional imaging is costly and is not a routine screening procedure. ${ }^{20}$ Consequently, more sensitive and more specific detection assays are needed that avoid unnecessary additional imaging and surgery from initial false-positive mammographic results. miRNA analysis of blood or other body fluids offers an inexpensive and noninvasive screening approach to more thoroughly examine high-risk individuals, either those with genetic predispositions or post-treatment patients at risk of recurrence.

\section{miRNA biomarkers in blood}

miRNAs are promising blood biomarkers because cell-free miRNA molecules that are circulating unaccompanied, associated with protein complexes, or encapsulated in membranebound vesicles (eg, exosome and microvesicles) are very stable in blood. ${ }^{21,22}$ However, circulating miRNAs may emanate from 
Table 3 miRNA signatures for prognosis and treatment response in $\mathrm{ER}^{+}$breast cancer subtypes

\begin{tabular}{|c|c|c|c|c|c|}
\hline miRNA(s) & Patient cohort & Sample & Methodology & Clinical observation(s) & Reference \\
\hline let-7b & 2,033 cases $\left(\mathrm{ER}^{+}[84 \%]\right.$ vs $\left.\mathrm{ER}^{-}[16 \%]\right)$ & $\begin{array}{l}\text { FFPE } \\
\text { tissue } \\
\text { cores }\end{array}$ & In situ hybridization & $\begin{array}{l}\text { Higher levels of let-7b } \\
\text { correlate with better } \\
\text { outcome in } \mathrm{ER}^{+} \text {cases. }\end{array}$ & 163 \\
\hline $\begin{array}{l}\text { miR-7, miR-128a, } \\
\text { miR-2I0, miR-516-3p }\end{array}$ & I47 early-stage $\mathrm{ER}^{+}$cases with $\mathrm{LN}^{-}$ & $\begin{array}{l}\text { FFPE } \\
\text { tissue }\end{array}$ & $\begin{array}{l}\text { TaqMan qRT-PCR } \\
\text { (Thermo Fisher Scientific) }\end{array}$ & $\begin{array}{l}\text { Correlates with shorter } \\
\text { time to distant metastasis. }\end{array}$ & 65 \\
\hline miR-10a, miR-126 & $\begin{array}{l}\text { Training set: I } 2 \text { early-stage } \mathrm{ER}^{+} \text {cases } \\
\left(\mathrm{LN}^{-}[83.3 \%] \text { vs } \mathrm{LN}^{+}[16.7]\right) \\
\text { Validation set: } 8 \text { I } \mathrm{ER}^{+} \text {cases } \\
\text { (Stage I-II [77.5\%] vs Stage III [23.5\%], } \\
\mathrm{LN}^{-}[46.9 \%] \text { vs } \mathrm{LN}^{+}[5 \mathrm{I} .8 \%] \text { ) treated } \\
\text { with tamoxifen monotherapy }\end{array}$ & $\begin{array}{l}\text { FFPE } \\
\text { tissue }\end{array}$ & $\begin{array}{l}\text { TaqMan qRT-PCR } \\
\text { (Thermo Fisher Scientific) }\end{array}$ & $\begin{array}{l}\text { Predicts response to } \\
\text { tamoxifen and correlates } \\
\text { with longer recurrence- } \\
\text { free survival. }\end{array}$ & 60 \\
\hline miR-19a, miR-205 & $\begin{array}{l}68 \text { luminal } A^{a} \text { cases (Stage II [16.2\%] vs } \\
\text { Stage III }[83.8 \%]) \text { treated with } \\
\text { neoadjuvant epirubicin + paclitaxel }\end{array}$ & Serum & $\begin{array}{l}\text { SYBR-based qRT-PCR } \\
\text { (Quantobio Technology) }\end{array}$ & $\begin{array}{l}\text { Predicts response to } \\
\text { epirubicin + paclitaxel. }\end{array}$ & 118 \\
\hline miR-30c & $\begin{array}{l}246 \text { advanced-stage } E^{+} \text {cases (local } \\
\text { recurrence }[13 \%] \text { vs distant recurrence } \\
{[87 \%] \text { ) treated with tamoxifen }}\end{array}$ & $\begin{array}{l}\text { FFPE } \\
\text { tissue }\end{array}$ & $\begin{array}{l}\text { TaqMan qRT-PCR } \\
\text { (Thermo Fisher Scientific) }\end{array}$ & $\begin{array}{l}\text { Predicts response to } \\
\text { tamoxifen and correlates } \\
\text { with longer progression- } \\
\text { free survival. }\end{array}$ & 61 \\
\hline $\operatorname{miR}-210$ & $\begin{array}{l}89 \text { early-stage } \mathrm{ER}^{+} \text {cases }\left(\mathrm{LN}^{-}[56 \%] \text { vs }\right. \\
\left.\mathrm{LN}^{+}[38 \%]\right) \text { treated with adjuvant } \\
\text { tamoxifen monotherapy }\end{array}$ & $\begin{array}{l}\text { FFPE } \\
\text { tissue }\end{array}$ & $\begin{array}{l}\text { TaqMan qRT-PCR } \\
\text { (Thermo Fisher Scientific) }\end{array}$ & $\begin{array}{l}\text { Correlates with shorter } \\
\text { recurrence-free survival. }\end{array}$ & 62 \\
\hline $\operatorname{miR}-519 a$ & $50 \mathrm{ER}^{+}$cases & $\begin{array}{l}\text { FFPE } \\
\text { tissue }\end{array}$ & $\begin{array}{l}\text { TaqMan qRT-PCR } \\
\text { (Thermo Fisher Scientific) }\end{array}$ & $\begin{array}{l}\text { Correlates with shorter } \\
\text { recurrence-free survival. }\end{array}$ & 63 \\
\hline
\end{tabular}

Notes: aLuminal A subtype was defined by expression of ER and/or PR, absence of HER2 expression, and less than $14 \%$ of cells positive for Ki-67.

Abbreviations: ER, estrogen receptor; FFPE, formalin-fixed paraffin-embedded; LN, lymph node status; miRNA, microRNA; PR, progesterone receptor; HER2, human EGF-like receptor 2; qRT-PCR, quantitative real-time polymerase chain reaction.

different cell types in the primary tumor lesion or systemically, and reflect: 1) the number of lysed cancer cells or other cells in the tumor microenvironment, 2) the number of cells expressing and secreting those particular miRNAs, and/or 3) the number of cells mounting an inflammatory or other physiological response against diseased tissue. Ideally for analysis, circulating miRNAs would reflect the number of cancer cells or other cell types specific to breast cancer in the primary tumor. Many studies have compared changes in miRNA levels in blood between breast cancer cases and age-matched healthy controls in order to identify miRNA biomarkers (Table 1). Unfortunately, there is significant variability among studies in the patient characteristics, experimental design, sample preparation, and detection methodology that complicates the interpretation of these studies:

- Patient characteristics: Clinical and pathological characteristics of patients and their tumor tissues differ broadly. Age, ethnicity, stage, histology, molecular subtype, and treatment history are variables that can affect miRNA expression. $^{23}$

Table 4 miRNA signatures for prognosis and treatment response in $\mathrm{HER}^{+}$breast cancer subtypes

\begin{tabular}{|c|c|c|c|c|c|}
\hline miRNA(s) & Patient cohort & Sample & Methodology & Clinical observation(s) & Reference \\
\hline miR-2I & $\begin{array}{l}32 \text { Stage III HER2 } 2^{+} \text {cases } \\
\left(\mathrm{ER}^{+}[56.2 \%] \text { vs } \mathrm{ER}^{-}[43.8 \%]\right)\end{array}$ & $\begin{array}{l}\text { Frozen tissues (pre- } \\
\text { and post-neoadjuvant } \\
\text { treatment) }\end{array}$ & $\begin{array}{l}\text { TaqMan qRT-PCR } \\
\text { (Thermo Fisher } \\
\text { Scientific) }\end{array}$ & $\begin{array}{l}\text { Higher levels correlate } \\
\text { with poor treatment } \\
\text { response. }\end{array}$ & 79 \\
\hline $\begin{array}{l}\operatorname{miR}-21 \\
\operatorname{miR}-210 \\
\operatorname{miR}-373\end{array}$ & $\begin{array}{l}\text { I } 27 \mathrm{HER}^{+} \text {cases (ER+ }[56 \%] \text { vs ER } \mathrm{ER}^{-}[44 \%] \\
\mathrm{LN}^{-}[40 \%] \text { vs } \mathrm{LN}^{+}[60 \%] ; \text { M0 }[84 \%] \text { vs } \\
\text { MI }[16 \%]) \text { with neoadjuvant treatment } \\
\text { (trastuzumab }[50 \%] \text { vs lapatinib }[50 \%] \text { ) }\end{array}$ & $\begin{array}{l}\text { Serum (pre- and } \\
\text { post-neoadjuvant } \\
\text { treatment) }\end{array}$ & $\begin{array}{l}\text { TaqMan qRT-PCR } \\
\text { (Thermo Fisher } \\
\text { Scientific) }\end{array}$ & $\begin{array}{l}\text { No correlation with } \\
\text { pathologic complete } \\
\text { response. High levels } \\
\text { of miR-2 I correlate } \\
\text { with overall survival. }\end{array}$ & 120 \\
\hline miR-2I0 & $\begin{array}{l}29 \mathrm{HER}^{+} \text {cases }\left(\mathrm{ER}^{+}[44.8 \%] \mathrm{vs}\right. \\
\mathrm{ER}^{-}[55.2 \%] ; \mathrm{LN}^{-}[34.4 \%] \mathrm{vs} \mathrm{LN} \mathrm{LN}^{+}[65.6 \%] ; \\
\text { with neoadjuvant treatment } \\
\text { (trastuzumab + chemotherapy) }\end{array}$ & $\begin{array}{l}\text { Plasma (pre- and } \\
\text { post-neoadjuvant } \\
\text { treatment) }\end{array}$ & $\begin{array}{l}\text { TaqMan qRT-PCR } \\
\text { (Thermo Fisher } \\
\text { Scientific) }\end{array}$ & $\begin{array}{l}\text { Higher circulating levels } \\
\text { correlate with pathologic } \\
\text { complete response, tumor } \\
\text { presence, and } \mathrm{LN}^{+} \text {status. }\end{array}$ & 119 \\
\hline
\end{tabular}

Abbreviations: ER, estrogen receptor; HER2, human EGF-like receptor 2; miRNA, microRNA; LN, lymph node status; qRT-PCR, quantitative real-time polymerase chain reaction. 
Table 5 miRNA signatures for prognosis and treatment response in TNBC subtype

\begin{tabular}{|c|c|c|c|c|c|}
\hline miRNA(s) & Patient cohort & Sample & Methodology & Clinical observation(s) & Reference \\
\hline $\begin{array}{l}\text { miR-I0b, miR-2 I, } \\
\text { miR-I22a, miR-I45, } \\
\text { miR-205, miR-2 I } 0\end{array}$ & 49 TNBC cases & $\begin{array}{l}\text { FFPE } \\
\text { tissues }\end{array}$ & $\begin{array}{l}\text { SYBR green qRT-PCR } \\
\text { (Qiagen NV) }\end{array}$ & $\begin{array}{l}\text { Correlates with shorter } \\
\text { disease-free and overall } \\
\text { survival. }\end{array}$ & 164 \\
\hline $\begin{array}{l}\text { miR-I0b-5p, miR-2I-3p, } \\
\text { miR-3I-5p, miR-I25b-5p, } \\
\text { miR-I30a-3p, miR-155-5p, } \\
\text { miR-18Ia-5p, miR-18Ib-5p, } \\
\text { miR-183-5p, miR-195-5p, } \\
\text { miR-45Ia }\end{array}$ & I5 TNBC cases & $\begin{array}{l}\text { Fresh } \\
\text { tissues }\end{array}$ & $\begin{array}{l}\text { SYBR green qRT-PCR } \\
\text { (Takara Bio Inc.) }\end{array}$ & $\begin{array}{l}\text { Separates TNBC tissues } \\
\text { from normal breast tissue. } \\
\text { Signature enriched for } \\
\text { miRNAs involved in } \\
\text { chemoresistance. }\end{array}$ & 86 \\
\hline $\begin{array}{l}\text { miR-I6, miR-I25b, } \\
\text { miR-I 55, miR-374a }\end{array}$ & $\begin{array}{l}\text { I73 TNBC cases }\left(\mathrm{LN}^{-}[35.8 \%]\right. \\
\left.\text { vs } \mathrm{LN}^{+}[64.2 \%]\right)\end{array}$ & $\begin{array}{l}\text { FFPE } \\
\text { tissues }\end{array}$ & NanoString nCounter & $\begin{array}{l}\text { Correlates with shorter } \\
\text { overall survival. }\end{array}$ & 165 \\
\hline miR-2I & $\begin{array}{l}72 \text { TNBC cases (Stage I-II } \\
\text { [45.8\%] vs Stage III-IV [54.2\%]; } \\
\left.\mathrm{LN}^{-}[5 \mathrm{I} .3 \%] \text { vs } \mathrm{LN}^{+}[48.6 \%]\right)\end{array}$ & $\begin{array}{l}\text { Frozen } \\
\text { tissues }\end{array}$ & $\begin{array}{l}\text { SYBR green QRT-PCR } \\
\text { (Thermo Fisher } \\
\text { Scientific) }\end{array}$ & $\begin{array}{l}\text { Correlates with shorter } \\
\text { recurrence-free survival. }\end{array}$ & 166 \\
\hline & $\begin{array}{l}\text { I05 early-stage TNBC cases } \\
\text { (Stage I [48.5\%] vs Stage II } \\
{[51.5 \%] ; \mathrm{LN}^{-}[67.6 \%] \text { vs }} \\
\left.\mathrm{LN}^{+}[32.4 \%]\right)\end{array}$ & $\begin{array}{l}\text { FFPE } \\
\text { tissue } \\
\text { cores }\end{array}$ & In situ hybridization & $\begin{array}{l}\text { High levels in stroma } \\
\text { compartment correlate with } \\
\text { shorter recurrence-free and } \\
\text { breast cancer-specific survival. }\end{array}$ & 97 \\
\hline $\begin{array}{l}\text { miR-27a, miR-30e, } \\
\text { miR-155, miR-493 }\end{array}$ & $\begin{array}{l}\text { I } 73 \text { TNBC cases ( } \mathrm{LN}^{-}[35.8 \%] \text { vs } \\
\left.\mathrm{LN}^{+}[64.2 \%]\right)\end{array}$ & $\begin{array}{l}\text { FFPE } \\
\text { tissues }\end{array}$ & NanoString nCounter & $\begin{array}{l}\text { Divides cases into risk } \\
\text { subgroups. }\end{array}$ & 84 \\
\hline $\begin{array}{l}\text { miR-27b, miR-150, } \\
\text { miR-342 }\end{array}$ & 37 TNBC cases & $\begin{array}{l}\text { Frozen } \\
\text { tissues }\end{array}$ & $\begin{array}{l}\text { Illumina miRNA } \\
\text { arrays }\end{array}$ & $\begin{array}{l}\text { Correlates with shorter } \\
\text { recurrence-free survival. }\end{array}$ & 67 \\
\hline $\begin{array}{l}\text { miR-190a, miR-200b-3p, } \\
\text { miR-5I2-5p }\end{array}$ & $\begin{array}{l}\text { Eleven TNBC cases (Stage I-II } \\
{[36.3 \%] \text { vs Stage III-IV [63.7\%]; }} \\
\left.\text { LN }^{-}[27.2 \%] \text { vs LN } \text { LN }^{+}[72.8 \%]\right) \\
\text { treated with different neoadjuvant } \\
\text { chemotherapy regimens }\end{array}$ & $\begin{array}{l}\text { Tissue } \\
\text { core } \\
\text { biopsies }\end{array}$ & $\begin{array}{l}\text { SYBR green } \mathrm{QRT}-\mathrm{PCR} \\
\text { (Exiqon) }\end{array}$ & $\begin{array}{l}\text { Predicts response } \\
\text { to treatment. }\end{array}$ & 85 \\
\hline miR-34b & $\begin{array}{l}39 \text { TNBC cases (Stage I-II [80\%] vs } \\
\text { Stage III-IV [20\%]; LN } \text { LN }^{-}[44 \%] \text { vs } \\
\left.\mathrm{LN}^{+}[56 \%]\right)\end{array}$ & $\begin{array}{l}\text { FFPE } \\
\text { tissues }\end{array}$ & $\begin{array}{l}\text { TaqMan qRT-PCR } \\
\text { (Thermo Fisher } \\
\text { Scientific) }\end{array}$ & $\begin{array}{l}\text { Correlates with shorter } \\
\text { disease-free and overall } \\
\text { survival. }\end{array}$ & 167 \\
\hline miR-200c, miR-205 & $\begin{array}{l}32 \text { TNBC cases }\left(\mathrm{LN}^{-}[50 \%] \text { vs }\right. \\
\left.\mathrm{LN}^{+}[50 \%]\right)\end{array}$ & $\begin{array}{l}\text { FFPE } \\
\text { tissues }\end{array}$ & $\begin{array}{l}\text { SYBR green } \text { QRT-PCR } \\
\text { (Qiagen NV) }\end{array}$ & $\begin{array}{l}\text { Lower levels correlate } \\
\text { with } \mathrm{LN}^{+} \text {status. }\end{array}$ & 168 \\
\hline \multirow[t]{2}{*}{ miR-2 I 0} & $\begin{array}{l}\text { II } 4 \text { early-stage } \mathrm{ER}^{-} \text {cases } \\
\text { with } \mathrm{LN}^{-} \text {status }\end{array}$ & $\begin{array}{l}\text { FFPE } \\
\text { tissues }\end{array}$ & $\begin{array}{l}\text { TaqMan qRT-PCR } \\
\text { (Thermo Fisher } \\
\text { Scientific) }\end{array}$ & $\begin{array}{l}\text { Correlates with shorter } \\
\text { time to distant metastasis. }\end{array}$ & 65 \\
\hline & $\begin{array}{l}58 \text { TNBC cases }\left(\mathrm{LN}^{-}[68.9 \%] \text { vs }\right. \\
\left.\mathrm{LN}^{+}[29.3 \%]\right)\end{array}$ & $\begin{array}{l}\text { Frozen } \\
\text { tissues }\end{array}$ & $\begin{array}{l}\text { TaqMan qRT-PCR } \\
\text { (Thermo Fisher } \\
\text { Scientific) }\end{array}$ & $\begin{array}{l}\text { Correlates with shorter disease- } \\
\text { free and overall survival. }\end{array}$ & 66 \\
\hline $\begin{array}{l}\text { miR-376b, miR-38I, } \\
\text { miR-409-5p, miR-4I0, } \\
\text { miR-766 }\end{array}$ & II 4 TNBC cases & $\begin{array}{l}\text { Frozen } \\
\text { tissues }\end{array}$ & $\begin{array}{l}\text { miRNA arrays } \\
\text { (Agilent } \\
\text { Technologies) }\end{array}$ & $\begin{array}{l}\text { Correlates with shorter distant } \\
\text { metastasis-free and breast } \\
\text { cancer-specific survival. }\end{array}$ & 169 \\
\hline
\end{tabular}

Note: microRNAs in bold show a recurrent presence in at least three independent studies.

Abbreviations: FFPE, formalin-fixed paraffin-embedded; LN, lymph node status; TNBC, triple-negative breast cancer; miRNA, microRNA; qRT-PCR, quantitative real-time polymerase chain reaction.

- Experimental design: Sample size and the inclusion of training and validation sets vary. Some studies analyzed changes in miRNA levels between fewer than 30 breast cancer and 30 control samples in a single patient cohort, whereas others analyzed these changes in much larger patient cohorts and validated miRNA signatures using independent cohorts. Such differences affect the statistical power of analysis. The miRNA field must be aware of the pitfalls associated with small sample sizes, poor experimental design, and statistical choices. ${ }^{24}$
- Sample preparation: Whole blood, serum, and plasma have been used as sample material for miRNA detection. Whole blood contains various cell types (white cells, red cells, and platelets) that contribute their miRNA content to the sample being analyzed, confounding interpretation of results. For this reason, serum or plasma are preferred sources of circulating miRNAs. Serum is obtained after blood coagulation and contains the liquid portion of blood with its proteins and other soluble molecules, but without cells or clotting factors. Plasma is obtained from 
Table 6 miRNA signatures for detection, monitoring, and characterization of MBC

\begin{tabular}{|c|c|c|c|c|c|}
\hline microRNA(s) & Patient cohort & Sample & Methodology & Clinical observation & Reference \\
\hline \multirow[t]{6}{*}{ miR-IOb } & $\begin{array}{l}23 \text { cases (M0 [2I.7\%] vs } \\
\text { MI }[78.3 \%])\end{array}$ & FFPE tissues & $\begin{array}{l}\text { SYBR green qRT-PCR } \\
\text { (Thermo Fisher Scientific) }\end{array}$ & Higher levels in MBC cases. & 100 \\
\hline & $\begin{array}{l}\text { I0I cases }\left(\mathrm{ER}^{+}[62.4 \%] \text { vs }\right. \\
\mathrm{ER}^{-} \text {cases }[37.6 \%] ; \mathrm{LN}^{-}[33.7 \%] \text { vs } \\
\mathrm{LN}^{+}[66.3 \%] ; \text { Stage I-II } \\
[59.4 \%] \text { vs Stage III-IV }[40.6 \%])\end{array}$ & FFPE tissues & $\begin{array}{l}\text { TaqMan qRT-PCR } \\
\text { (Thermo Fisher Scientific) }\end{array}$ & $\begin{array}{l}\text { Higher levels in MBC cases; higher } \\
\text { levels correlate with shorter } \\
\text { progression-free and overall } \\
\text { survival in metastasis-free cases. }\end{array}$ & 102 \\
\hline & $\begin{array}{l}84 \text { early-stage cases }\left(\mathrm{ER}^{+}[53.6 \%] \text { vs }\right. \\
\mathrm{ER}^{-} \text {cases }[41.1 \%] ; \mathrm{LN}^{-}[24.1 \%] \text { vs } \\
\left.\mathrm{LN}^{+}[75.9 \%]\right)\end{array}$ & FFPE tissues & $\begin{array}{l}\text { TaqMan qRT-PCR } \\
\text { (Thermo Fisher Scientific) }\end{array}$ & $\begin{array}{l}\text { No correlation with disease } \\
\text { progression, metastasis, } \\
\text { or clinical outcome. }\end{array}$ & 106 \\
\hline & $\begin{array}{l}219 \text { cases }\left(\mathrm{LN}^{-}[58 \%] \text { vs }\right. \\
\left.\mathrm{LN}^{+}[42 \%]\right)\end{array}$ & $\begin{array}{l}\text { Frozen } \\
\text { tissues }\end{array}$ & $\begin{array}{l}\text { TaqMan qRT-PCR } \\
\text { (Thermo Fisher Scientific) }\end{array}$ & $\begin{array}{l}\text { No correlation with formation } \\
\text { of distant metastasis or clinical } \\
\text { outcome. }\end{array}$ & 107 \\
\hline & $\begin{array}{l}\text { I } 22 \text { cases (M0 [82\%] vs } \\
\text { MI [I8\%]) and } 59 \text { age-matched } \\
\text { healthy controls }\end{array}$ & Serum & $\begin{array}{l}\text { SYBR green qRT-PCR } \\
\text { (Thermo Fisher Scientific) }\end{array}$ & $\begin{array}{l}\text { Higher levels in } M B C \text { cases } \\
\text { with bone metastases. }\end{array}$ & 104 \\
\hline & $\begin{array}{l}\text { I } 52 \text { cases (M0 [78.9\%] vs } \\
\text { MI }[2|.| \%]) \text { and } 40 \text { healthy } \\
\text { controls }\end{array}$ & $\begin{array}{l}\text { Serum (post- } \\
\text { surgery for } \\
\text { M0 cases) }\end{array}$ & $\begin{array}{l}\text { TaqMan qRT-PCR } \\
\text { (Thermo Fisher Scientific) }\end{array}$ & $\begin{array}{l}\text { No change in levels change } \\
\text { between non-MBC and } \\
M B C \text { cases. }\end{array}$ & 170 \\
\hline $\begin{array}{l}\text { miR-I Ob, } \\
\text { miR-373 }\end{array}$ & $\begin{array}{l}60 \text { cases }\left(\mathrm{ER}^{+}[60 \%] \text { vs } \mathrm{ER}^{-} \text {cases }\right. \\
{[40 \%] ; \mathrm{LN}^{-}[4 \mathrm{I} .7 \%] \text { vs }} \\
\mathrm{LN}^{+}[58.3 \%] ; \text { Stage I-II [\%]) }\end{array}$ & Plasma & $\begin{array}{l}\text { TaqMan qRT-PCR } \\
\text { (Thermo Fisher Scientific) }\end{array}$ & Higher levels in cases with $\mathrm{LN}^{+}$. & 103 \\
\hline $\begin{array}{l}\text { miR-17, } \\
\text { miR-I55 }\end{array}$ & $\begin{array}{l}\mathrm{I} 52 \text { cases (M0 [78.9\%] vs MI } \\
[21.1 \%]) \text { and } 40 \text { healthy controls }\end{array}$ & Serum & $\begin{array}{l}\text { TaqMan qRT-PCR } \\
\text { (Thermo Fisher Scientific) }\end{array}$ & $\begin{array}{l}\text { Levels change between } \\
\text { non-MBC and } M B C \text { cases. }\end{array}$ & 170 \\
\hline miR-19b & $\begin{array}{l}\text { II } 3 \text { cases (HER2- }[42.4 \%] \text { vs } \\
\text { HER2 } 2^{+}[57.5 \%] ; \text { M0 [3I\%] vs } \\
\text { MI }[69 \%] \text { ) and } 30 \text { age-matched } \\
\text { healthy controls }\end{array}$ & Serum & $\begin{array}{l}\text { TaqMan qRT-PCR } \\
\text { (Thermo Fisher Scientific) }\end{array}$ & $\begin{array}{l}\text { Correlates with longer overall } \\
\text { survival in HER2 } 2^{+} \mathrm{MBC} \text { cases } \\
\text { with inflammatory disease. }\end{array}$ & 105 \\
\hline $\begin{array}{l}\text { miR-2I, } \\
\text { miR-205 }\end{array}$ & $\begin{array}{l}84 \text { early-stage cases }\left(\mathrm{ER}^{+}[53.6 \%] \text { vs }\right. \\
\mathrm{ER}^{-} \text {cases }[41.1 \%] ; \mathrm{LN}^{-}[24.1 \%] \text { vs } \\
\left.\mathrm{LN}^{+}[75.9 \%]\right)\end{array}$ & FFPE tissues & $\begin{array}{l}\text { TaqMan qRT-PCR } \\
\text { (Thermo Fisher Scientific) }\end{array}$ & $\begin{array}{l}\text { Correlates with shorter } \\
\text { recurrence-free survival. Only } \\
\text { lower levels of miR-205 correlate } \\
\text { with shorter overall survival. }\end{array}$ & 106 \\
\hline miR-2I0 & $\begin{array}{l}219 \text { cases }\left(\mathrm{LN}^{-}[58 \%] \text { vs }\right. \\
\left.\mathrm{LN}^{+}[42 \%]\right)\end{array}$ & $\begin{array}{l}\text { Frozen } \\
\text { tissues }\end{array}$ & $\begin{array}{l}\text { TaqMan qRT-PCR } \\
\text { (Thermo Fisher Scientific) }\end{array}$ & $\begin{array}{l}\text { Higher levels correlate with } \\
\text { shorter recurrence-free survival. }\end{array}$ & 107 \\
\hline miR-I258 & $\begin{array}{l}\text { I } 66 \mathrm{BC} \text { cases (M0 [48.7\%] vs MI } \\
\text { [5I.3\%]), } 62 \text { cases with benign } \\
\text { breast disease and } 54 \text { healthy } \\
\text { controls }\end{array}$ & $\begin{array}{l}\text { Serum (post- } \\
\text { surgery } \\
\text { but before } \\
\text { treatment) }\end{array}$ & $\begin{array}{l}\text { SYBR green qRT-PCR } \\
\text { (Shanghai Novland } \\
\text { Co. Ltd) }\end{array}$ & $\begin{array}{l}\text { Lower circulating levels in BMC } \\
\text { cases compared to non-BMC } \\
\text { cases and healthy controls. } \\
\text { Higher circulating levels correlate } \\
\text { with good clinical outcome. }\end{array}$ & $17 \mid$ \\
\hline
\end{tabular}

Note: microRNAs in bold show a recurrent presence in at least three independent studies.

Abbreviations: BC, breast cancer; ER, estrogen receptor; FFPE, formalin-fixed paraffin-embedded; LN, lymph node status; MBC, metastatic breast cancer; miRNA, microRNA; HER2, human EGF-like receptor 2; qRT-PCR, quantitative real-time polymerase chain reaction.

uncoagulated blood; it contains the liquid portion of blood with clotting factors, proteins, and molecules not present in serum, but it also retains some cells. Additionally, different anticoagulants can be used to prepare plasma (eg, heparin and ethylenediaminetetraacetic acid [EDTA]), and these can have different effects on plasma composition and downstream molecular assays. The lysis of red blood cells or other cell types (hemolysis) during blood separation procedures can contaminate the miRNA content in serum and plasma preparations. Several miRNAs are known to be expressed at high levels in specific blood cell types, and these miRNAs are typically excluded from analysis to avoid confusion.
Moreover, it appears that miRNA concentration in serum is higher than in plasma, hindering direct comparison of studies using these different starting materials. ${ }^{25}$

- Detection methodology: The miRCURY LNA Universal RT miRNA and PCR assay, and the TaqMan Low Density Array RT-PCR assay are among the most frequently used high-throughput RT-PCR platforms for miRNA detection. Each uses a different strategy to reverse transcribe mature miRNA molecules and to PCR-amplify the cDNA, which results in different detection biases.

- Data analysis: One of the biggest challenges to date is the normalization of circulating miRNA levels. Since 
there is not a unique cellular source or mechanism by which miRNAs reach circulation, choosing a reference miRNA (eg, miR-16, miR-26a) or other non-coding RNA (eg, U6 snRNA, snoRNA RNU43) is not straightforward. Spiking samples with RNA controls and/or normalization of miRNA levels to volume are some of the strategies used to standardize analysis. In addition, various studies apply different statistical methods and criteria for normalization, background or control reference subtraction, and significance cutoff values. ${ }^{12}$

Due to this variability in assay methods and analysis, it is not surprising that the reported signatures present little overlap. If one focuses on common trends, there are some miRNAs that might be useful for early detection of all types of breast cancer, whereas others might be useful for specific subtypes, histologies, or disease stages (Table 1). We briefly describe recent studies that used previous works to inform their experimental approach and analysis. Leidner et al drew and harmonized miRNA data from 15 previous studies and compared circulating miRNA signatures. ${ }^{26}$ They found very few miRNAs whose changes in circulating levels between breast cancer and control samples were consistent even when using similar detection methods (mainly quantitative real-time polymerase chain reaction [qRT-PCR] assays). There was no consistency at all between circulating miRNA signatures generated using different genome-wide detection platforms after filtering out contaminating miRNAs from cellular sources in the blood. The authors then performed their own study that included plasma samples from 20 breast cancer patients before surgery, 20 age- and racematched healthy controls, an independent set of 20 breast cancer patients after surgery, and ten patients with lung or colorectal cancer. Forty-six circulating miRNAs showed significant changes between pre-surgery breast cancer patients and healthy controls. Using other reference groups in the study, the authors could assign miRNA changes to different categories. The change in the circulating amount of 13 of these miRNAs was similar between post-surgery breast cancer cases and healthy controls, suggesting that the changes in these miRNAs in pre-surgery patients reflected the presence of a primary breast cancer tumor. ${ }^{26}$ However, ten of the 13 miRNAs also showed altered plasma levels in patients with other cancer types, suggesting that they may more generally reflect a tumor presence or tumor burden. After these analyses, only three miRNAs (miR-92b*, miR568 , and $\mathrm{miR}-708^{*}$ ) were identified as breast cancer-specific circulating miRNAs. These miRNAs had not been identified in previous studies.
More recently, Shen et al found 43 miRNAs that were detected at significantly different levels in plasma samples from a training set of 52 patients with invasive breast cancer, 35 with noninvasive ductal carcinoma in situ (DCIS), and 35 healthy controls, ${ }^{27}$ all study subjects were Caucasian. miR-33a, miR-136, and miR-199-a5-p were among those with the highest fold change between invasive carcinoma cases and healthy controls or DCIS cases. These changes in circulating miRNA levels may reflect advanced malignancy events. Twenty-three miRNAs exhibited consistent changes between invasive carcinoma and DCIS cases relative to healthy controls, which may reflect early malignancy changes. Interestingly, only three of these 43 miRNAs overlapped with miRNAs in previously reported signatures. These three, miR-133a, miR-148b, and miR-409-3p, were all part of the early malignancy signature and their fold changes were relatively modest, less than four-fold. Nonetheless, the authors validated the changes of miR-133a and miR-148b in plasma samples from an independent cohort of 50 patients with stage I and II breast cancer and 50 healthy controls. Moreover, miR-133a and miR-148b were detected in culture media of MCF-7 and MDA-MB-231 cells, suggesting that they are secreted by the cancer cells. The authors did not investigate the mechanism of miRNA secretion.

Some studies have also compared changes in the amount of circulating miRNAs in blood samples obtained before or after surgery (Table 1). A four-miRNA signature (miR-107, miR-148a, miR-223, and miR-338-3p) was identified in a patient cohort of $24 \mathrm{ER}^{+}$breast cancers. ${ }^{28}$ Circulating serum levels of miR-148a, miR-223, and miR-338-3p decreased, while that of miR-107 increased after surgery. ${ }^{28}$ Normalization of circulating miRNA levels after surgery could be useful in detecting disease recurrence if the changes are also observed in blood samples collected during follow-up visits. In another study, circulating levels of miR-19a, miR-24, miR-155, and miR-181b were monitored longitudinally in serum samples from a cohort of 63 breast cancer patients collected 1 day before surgery, 2-4 weeks after surgery, and 2-4 weeks after the first cycle of adjuvant treatment. ${ }^{29}$ Levels of miR-24, miR-155, and miR-181b decreased after surgery, while the level of miR-19a only significantly decreased after adjuvant treatment. ${ }^{29}$ The authors noted that three patients relapsed during the study follow-up. This limited number did not allow the authors to determine whether the altered levels of these miRNAs could be useful for detecting disease recurrence. ${ }^{29}$

The lack of consensus about circulating miRNA signatures for early detection of primary or recurrent breast tumor requires 
careful and thoughtful examination. Does this mainly indicate technical difficulties in preanalytic sample preparation, miRNA detection, and/or statistical analysis? Or does it more deeply question the validity of miRNAs as biomarkers for detecting a wide array of heterogeneous presentations of breast cancer? Longitudinal studies that collect blood from breast cancer patients, ideally before diagnosis (healthy baseline), at diagnosis, before surgery, and after surgery, that also consistently process and analyze miRNA changes should be considered to address these questions. High-risk individuals, such as $B R C A$ gene mutation carriers, those with other genetic predispositions to breast cancer, or breast cancer survivors at high risk of recurrence, could provide cohorts of appropriate size for such longitudinal studies.

Finally, detection of miRNAs within isolated exosomes or microvesicles is a potential new biomarker assay to consider. ${ }^{21,22}$ Enrichment of miRNAs in these membrane-bound particles may more directly reflect the secretory phenotype of cancer cells or other cells in the tumor microenvironment, than circulating miRNAs in whole blood samples. Such miRNAs may be less subject to noise and inter-patient variability, and thus may be a more appropriate material for analysis in longitudinal studies.

\section{Risk alleles of miRNA or target genes associated with breast cancer}

By mining the genome for allele variants of miRNA genes or their known target genes, miRNA research has shown some promise in helping identify individuals at risk of developing breast cancer. Single nucleotide polymorphisms (SNPs) in the miRNA precursor hairpin can affect its stability, miRNA processing, and/or altered miRNA-target mRNA binding interactions if the SNPs are within the functional sequence of mature miRNAs. Similarly, SNPs in the $3^{\prime}$-UTR of mRNAs can decrease or increase binding interactions with miRNA, altering protein expression. In addition, SNPs in coding sequences of proteins involved in miRNA processing (eg, DROSHA), export (eg, XPO5), and maturation (eg, Dicer) can also affect the expression levels and activity of miRNAs (Table 2). Depending on the tumor suppressive or oncogenic functions of a protein, disruption of miRNA-mediated regulation can increase or decrease cancer risk. According to the miRdSNP database, there are currently 14 unique genes experimentally confirmed as miRNA targets with breast cancer-associated SNPs in their 3'-UTRs (APC, BMPR1B, BRCA1, CCND1, CXCL12, CYP1B1, ESR1, IGF1, IGF1R, IRS2, PTGS2, SLC4A7, $T G F B R 1$, and $V E G F A) .{ }^{30}$ Table 2 provides a comprehensive summary of miRNA-related SNPs linked to breast cancer; some well-studied SNPs are highlighted below.

SNPs in the precursors of five miRNAs (miR-27a, miR146a, miR-149, miR-196, and miR-499) have been associated with increased risk of developing certain types of cancer, including breast cancer. ${ }^{31}$ Race, ethnicity, and molecular subtype can influence the relative risk associated with SNPs. ${ }^{32,33}$ The rare [G] allele of rs895819 is located in the loop of premiR-27; it interferes with miR-27 processing and is associated with a lower risk of developing familial breast cancer. ${ }^{34}$ The same allele was associated with lower risk of sporadic breast cancer in a patient cohort of young Chinese women, ${ }^{35}$ but the allele had no prognostic value in individuals with breast cancer in this cohort. ${ }^{35}$ The [C] allele of rs 11614913 in the pre-miR-196 and [G] allele of rs3746444 in the premiR-499 were associated with increased risk of developing breast cancer in a case-control study of Chinese women (1,009 breast cancer patients and 1,093 healthy controls). ${ }^{36}$ In contrast, the same variant alleles were not associated with increased breast cancer risk in a case-control study of Italian and German women (1,894 breast cancer cases and 2,760 healthy controls). ${ }^{37}$ The [C] allele of rs462480 and [G] allele of rs1053872, within $61 \mathrm{bp}$ and $10 \mathrm{~kb}$ of pre-miR-101, were associated with increased breast cancer risk in a casecontrol study of Chinese women (1,064 breast cancer cases and 1,073 healthy controls). ${ }^{38}$ The authors suggest that these SNPs may interfere with stability or processing of primary miRNA transcripts. ${ }^{38}$

The [G] allele of rs61764370 in the $3^{\prime}$-UTR of $K R A S$, which disrupts a binding site for let-7 family members, is associated with an increased risk of developing certain types of cancer, including breast cancer. The [G] allele of rs61764370 was associated with the TNBC subtype in younger women in case-control studies from Connecticut, US cohort with 415 breast cancer cases and 475 healthy controls, as well as from an Irish cohort with 690 breast cancer cases and 360 healthy controls. ${ }^{39}$ This allele was also associated with familial BRCA1 breast cancer in a casecontrol study with 268 mutated BRCA1 families, 89 mutated BRCA2 families, 685 non-mutated BRCA1/2 families, and 797 geographically matched healthy controls. ${ }^{40}$ However, there was no association between ER status and this allele in this study cohort. ${ }^{40}$ No association between this allele and the TNBC subtype or BRCA1 mutation status was found in an independent case-control study with 530 sporadic postmenopausal breast cancer cases, 165 familial breast cancer cases (regardless of BRCA status), and 270 postmenopausal healthy controls. ${ }^{41}$ 
Interestingly, the [C] allele of rs 7963551 in the $3^{\prime}$-UTR of RAD52 also disrupts a binding site for let-7. This allele is associated with decreased breast cancer risk in two independent case-control studies of Chinese women with 878 and 914 breast cancer cases and 900 and 967 healthy controls, respectively. ${ }^{42}$ The authors suggest that relief of let-7-mediated regulation may contribute to higher baseline levels of this DNA repair protein, which could be protective against cancer development. The [T] allele of rs 1434536 in the $3^{\prime}$-UTR of the bone morphogenic receptor type 1B (BMPR1B) disrupts a binding site for miR-125b. ${ }^{43}$ This variant allele was associated with increased breast cancer risk in a case-control study with 428 breast cancer cases and 1,064 healthy controls. ${ }^{43}$

\section{Prognostic and predictive miRNA biomarkers in breast cancer subtypes with targeted therapies}

$\mathrm{ER}^{+}$breast cancers account for $70 \%$ of all cases and have the best clinical outcome. For $\mathrm{ER}^{+}$cancers, several targeted therapies exist to block hormone signaling, including tamoxifen, aromatase inhibitors, and fulvestrant. However, as many as half of these patients are resistant to endocrine therapy intrinsically (de novo) or will develop resistance over time (acquired). ${ }^{44}$ Thus, there is a clinical need for prognostic and predictive biomarkers that can indicate which $\mathrm{ER}^{+}$patients can be effectively treated with hormone therapies alone and which tumors have innate (or will develop) resistance to hormone therapy, thereby requiring more aggressive treatment. For HER2 ${ }^{+}$breast cancers, treatment with the targeted inhibitor trastuzumab is the standard course. ${ }^{45,46}$ Even though trastuzumab is effective, almost half of the breast cancer patients that overexpress HER2 are either nonresponsive to trastuzumab or develop resistance. ${ }^{47-49}$ There have been numerous mechanisms identified for trastuzumab resistance, yet there is no clinical assay available to determine which patients will respond to trastuzumab.

Profiling of miRNA expression in clinical tissue specimens and/or in breast cancer cell line models of drug resistance has linked individual miRNAs or miRNA signatures to drug resistance and disease outcome (Tables 3 and 4). Functional characterization of some of the highlighted miRNAs in cell line models has provided mechanistic insights on their role in resistance. ${ }^{50,51}$ Some miRNAs can directly control expression levels of ER and HER2 via interaction with complementary binding sites on the $3^{\prime}$-UTRs of mRNAs. ${ }^{50,51}$ Other miRNAs can affect output of ER and HER2 signaling by controlling expression levels of downstream effectors and signaling factors. ${ }^{50,51}$

\section{miRNAs in ER signaling and endocrine resistance}

miR-22, miR-27a, miR-206, miR-221/222, and miR-302c have been shown to regulate ER expression in breast cancer cell line models and, in some instances, miRNA overexpression is sufficient to promote resistance to endocrine therapies. ${ }^{52-55}$ In some studies (but not others), these miRNAs have been detected at lower levels in $\mathrm{ER}^{+}$tumor tissues relative to ER $^{-}$tumor tissues. ${ }^{55,56}$ Expression of the miR-191 miR-425 gene cluster and of miR-342 is driven by ER signaling in breast cancer cell lines and their expression correlates with ER status in breast tumor tissues. ${ }^{56-59}$ Several clinical studies have identified individual miRNAs or miRNA signatures that correlate with response to adjuvant tamoxifen treatment. ${ }^{60-64}$ These signatures do not include any of the above-mentioned miRNAs that have a mechanistic link to ER regulation or signaling. A ten-miRNA signature (miR-139-3p, miR-190b, miR-204, miR-339-5p, miR-363, miR-365, miR-502-5p, $\mathrm{miR}-520 \mathrm{c}-3 \mathrm{p}, \mathrm{miR}-520 \mathrm{~g} / \mathrm{h}$, and miRPlus-E1130) was associated with clinical outcome in a patient cohort of $52 \mathrm{ER}^{+}$ cases treated with tamoxifen, but this signature could not be validated in two independent patient cohorts. ${ }^{64}$ Individual expression changes in miR-30c, miR-210, and miR-519 correlated with clinical outcome in independent patient cohorts treated with tamoxifen. ${ }^{60-63}$ High miR-210 correlated with shorter recurrence-free survival in a cohort of 89 patients with early-stage ER ${ }^{+}$breast tumors. ${ }^{62}$ The prognostic performance of miR-210 was comparable to that of mRNA signatures, including the 21-mRNA recurrence score from which US Food and Drug Administration (FDA)-cleared Oncotype Dx is derived. High miR-210 expression was also associated with poor outcome in other patient cohorts of either all comers or ER- cases. ${ }^{65-69}$ The expression of miR210 was also upregulated under hypoxic conditions. ${ }^{70}$ Thus, miR-210-based prognostic information may not be specific or limited to ER signaling or $\mathrm{ER}^{+}$breast tumors.

\section{miRNAs in HER signaling \\ and trastuzumab resistance}

miR-125b, miR-134, miR-193a-5p, miR-199b-5p, miR-331-3p, miR-342-5p, and miR-744* have been shown to regulate expression of HER2 via binding to sites on the $3^{\prime}$-UTR of its mRNA in HER2 ${ }^{+}$breast cancer cell lines (eg, BT-474, MDA-MB-453, and SK-BR-3). ${ }^{71-73} \mathrm{miR}-$ 125b and miR-205 also indirectly affect HER2 signaling 
via inhibition of HER3 in SK-BR-3 and MCF-7 cells. ${ }^{71,74}$ Expression of other miRNAs, including miR-26, miR-30b, and miR-194, is upregulated upon trastuzumab treatment in BT-474 and SK-BR-3 cells. ${ }^{75,76}$ Altered expression of these miRNAs has been associated with breast cancer, but for most of them, there is not a clear, exclusive link to the HER2 ${ }^{+}$tumor subtype. miR-21, miR-302f, miR-337, miR-376b, miR-520d, and miR-4728 have been reported by some studies (but not others) to be overexpressed in HER2 ${ }^{+}$breast cancer tissues. ${ }^{56,77,78}$ Indeed, miR-4728 is cotranscribed with the HER2 primary transcript and is processed out from an intronic sequence. ${ }^{78}$ High levels of miR-21 interfere with trastuzumab treatment in BT-474, MDA-MB-453, and SK-BR-3 cells via inhibition of PTEN (phosphatase and tensin homolog). ${ }^{79}$ High levels of miR-21 in HER2 ${ }^{+}$tumor tissues before and after neoadjuvant treatment with trastuzumab are associated with poor response to treatment. ${ }^{79} \mathrm{miR}-221$ can also confer resistance to trastuzumab treatment via PTEN in SK-BR-3 cells. ${ }^{80}$ High levels of miR-221 correlate with lymph node involvement and distant metastasis as well as HER2 overexpression, ${ }^{81}$ though other studies observed lower levels of miR-221 in HER2 ${ }^{+}$cases. $^{82}$

While these mechanistic interactions are sound and there are supportive data with clinical specimens, the prognostic value and potential clinical applications of these miRNAs are not clear. Future studies should investigate whether any of these miRNAs can inform disease outcome or treatment response within a more homogenous cohort of $\mathrm{HER}^{+}$cases.

\section{miRNA biomarkers and therapeutic opportunities in TNBC without targeted therapies}

TNBC is a highly heterogeneous disease whose clinical features include a peak risk of recurrence within the first 3 years, a peak of cancer-related deaths in the first 5 years, and a weak relationship between tumor size and lymph node metastasis. ${ }^{4}$ At the molecular level, TNBC has significant overlap with the basal-like subtype, with approximately $80 \%$ of TNBCs being classified as basal-like. ${ }^{3}$ A comprehensive gene expression analysis (mRNA signatures) of 587 TNBC cases revealed extensive molecular heterogeneity within TNBC as well as six distinct molecular TNBC subtypes. ${ }^{83}$ The molecular heterogeneity increases the difficulty of developing targeted therapeutics that will be effective in unstratified TNBC patients. It would be highly beneficial to be able to identify these molecular subtypes with simplified biomarkers or signatures.
miRNA expression profiling on frozen and fixed tissues using various detection methods have identified miRNA signatures or individual miRNA changes that correlate with clinical outcome in TNBC cases (Table 5). A four-miRNA signature (miR-16, miR-125b, miR-155, and miR-374a) correlated with shorter overall survival in a patient cohort of 173 TNBC cases. Reanalysis of this cohort by dividing cases into core basal (basal CK5/6- and/or epidermal growth factor receptor [EGFR]-positive) and 5NP (negative for all five markers) subgroups identified a different four-miRNA signature (miR-27a, miR-30e, miR-155, and miR-493) that correlated with the subgroup classification based on ER/ PR/HER2/basal cytokeratins/EGFR status. ${ }^{84}$ Accordingly, this four-miRNA signature can separate low- and high-risk cases - in some instances, even more accurately than core basal and 5NP subgroup stratification. ${ }^{84}$ Other miRNA signatures could be useful to inform treatment response to specific chemotherapy regimens (Table 5). A three-miRNA signature (miR-190a, miR-200b-3p, and miR-512-5p) obtained from tissue core biopsies before treatment correlated with complete pathological response in a limited patient cohort of eleven TNBC cases treated with different chemotherapy regimens. ${ }^{85}$ An eleven-miRNA signature (miR-10b, miR-21, miR-31, miR-125b, miR-130a-3p, miR-155, miR-181a, miR181b, miR-183, miR-195, and miR-451a) separated TNBC tumors from normal breast tissue. ${ }^{86}$ The authors noted that several of these miRNAs are linked to pathways involved in chemoresistance. ${ }^{86}$

Categorizing TNBC subgroups by gene expression (mRNA) signatures indicates the influence and contribution of stromal elements in driving and defining specific subgroups. ${ }^{83}$ Immunomodulatory, mesenchymal-like, and mesenchymal stem-like subtypes are characterized by signaling pathways typically carried out, respectively, by immune cells and stromal cells, including tumor-associated fibroblasts. miR10b, miR-21, and miR-155 are among the few miRNAs that are represented in multiple signatures found to be associated with poor outcome in TNBC. These miRNAs are known to be expressed in cell types other than breast cancer cells, ${ }^{87-91}$ and thus, their altered expression may reflect aberrant processes in the tumor microenvironment. ${ }^{92}$

In situ hybridization (ISH) assays are a powerful tool to determine altered miRNA expression at single-cell resolution and to assess the contribution of reactive stroma and immune response. ${ }^{13,93}$ In breast phyllodes tumors, ${ }^{94}$ as well as in colorectal ${ }^{95}$ and pancreatic cancer, ${ }^{96}$ upregulation of miR-21 expression promotes myofibrogenesis and regulates antimetastatic and proapoptotic target genes, including 
RECK (reversion-inducing cysteine-rich protein with kazal motifs), SPRY1/2 (Sprouty homolog 1/2 of Drosophila gene), PDCD-4 (programed cell death 4), and PTEN. We have recently shown that high levels of miR-21 expression in the stromal compartment in a cohort of 105 early-stage TNBC cases correlated with shorter recurrence-free and breast cancer-specific survival. ${ }^{97}$ While ISH-based miRNA detection is not as sensitive as that of a qRT-PCR assay, it provides an independent validation tool to determine the predominant cell type(s) that express miRNAs associated with TNBC or other breast cancer subtypes.

\section{miRNA biomarkers for monitoring and characterization of metastatic disease}

Although significant progress has been made in detecting and treating primary breast cancer, advances in the treatment of MBC have been marginal. Does molecular analysis of the primary tumor tissues reflect the evolution of metastatic lesions? Are we treating the wrong disease(s)? In the clinic, computed tomography (CT), positron emission tomography $(\mathrm{PET}) / \mathrm{CT}$, and magnetic resonance imaging (MRI) are conventional methods for monitoring $\mathrm{MBC}$ patients and evaluating therapeutic efficacy. However, these technologies are limited in their ability to detect microscopic lesions and immediate changes in disease progression. Because it is not currently standard practice to biopsy metastatic lesions to inform new treatment plans at distant sites, circulating tumor cells (CTCs) have been effectively used to evaluate disease progression and treatment response. CTCs represent the molecular composition of the disease and can be used as prognostic or predictive biomarkers to guide treatment options. Further advances have been made in evaluating tumor progression and response using circulating RNA and DNA in blood samples. miRNAs are promising markers that can be identified in primary and metastatic tumor lesions, as well as in CTCs and patient blood samples.

Several miRNAs, differentially expressed in primary tumor tissues, have been mechanistically linked to metastatic processes in cell line and mouse models. ${ }^{22,98}$ Most of these miRNAs are thought to exert their regulatory roles within the epithelial cell compartment (eg, miR-10b, miR-31, miR-141, miR-200b, miR-205, and miR-335), but others can predominantly act in other compartments of the tumor microenvironment, including tumor-associated fibroblasts (eg, miR-21 and miR-26b) and the tumor-associated vasculature (eg, miR-126). miR-10b has been more extensively studied than other miRNAs in the context of MBC (Table 6).
We briefly describe below some of the studies that have analyzed miR-10b in primary tumor tissues, as well as in blood from breast cancer cases with concurrent metastatic disease, either regional (lymph node involvement) or distant (brain, bone, lung).

miR-10b promotes invasion and metastatic programs in human breast cancer cell lines and mouse models via HoxD10 inhibition, which derepresses expression of the prometastatic gene $R h o C .^{99,100}$ In the original study, higher levels of miR-10b in primary tumor tissues correlated with concurrent metastasis in a patient cohort of five breast cancer cases without metastasis and $18 \mathrm{MBC}$ cases. ${ }^{100}$ Higher levels of miR-10b in the primary tumors correlated with concurrent brain metastasis in a cohort of $20 \mathrm{MBC}$ cases with brain metastasis and ten breast cancer cases without brain metastasis. ${ }^{101}$ In another study, miR-10b levels were higher in the primary tumors of $\mathrm{MBC}$ cases. ${ }^{102}$ Higher amounts of circulating miR-10b were also associated with cases having concurrent regional lymph node metastasis. ${ }^{103-105}$ However, another study on primary tumor tissues did not find an association between miR-10b levels and disease progression or clinical outcome in a cohort of 84 early-stage breast cancer patients ${ }^{106}$ or in another cohort of 219 breast cancer patients, ${ }^{107}$ both with long-term ( $>10$ years) clinical followup information.

We are not aware of any study that has compared miRNA expression between matched primary and metastatic tissues in a large cohort. This could provide information about cancer cell evolution, as well as the tumor microenvironment niche at distant sites. With smaller cohorts, higher levels of miR-9, miR-200 family members (miR-141, miR-200a, miR-200b, miR-200c), and miR-219-5p have been detected in distant metastatic lesions compared with matched primary tumors by RT-PCR and ISH assays. ${ }^{108}$

A recent ISH-based study in a limited number of breast cancer cases reported that expression of miR-708 was markedly downregulated in regional lymph node and distant lung metastases. ${ }^{109}$ miR-708 modulates intracellular calcium levels via inhibition of neuronatin. ${ }^{109}$ miR-708 expression is transcriptionally repressed epigenetically by polycomb repressor complex 2 in metastatic lesions, which leads to higher calcium bioavailability for activation of extracellular signal-regulated kinase (ERK) and focal adhesion kinase (FAK), and cell migration. ${ }^{109}$ Recent mechanistic studies have revealed antimetastatic functions of miR-7, ${ }^{110}$ miR-18a ${ }^{111}$ and miR-29b, ${ }^{112}$ as well as conflicting antimetastatic functions of miR-23 $\mathrm{b}^{113}$ and prometastatic functions of the miR-23 cluster (miR-23, miR-24, and miR-27b) ${ }^{114}$ in 
breast cancer. The prognostic value of these miRNAs needs to be investigated.

miRNA expression profiling in CTCs could be useful for assigning CTC status and for interrogating molecular aberrations in individual CTCs during the course of MBC. ${ }^{115}$ However, only one study has analyzed miRNA expression in CTC-enriched blood samples after positive selection of epithelial cells with anti-EpCAM antibody binding. ${ }^{116}$ The authors used a cutoff of 5 CTCs per $7.5 \mathrm{~mL}$ of blood to consider a sample positive for CTCs, which is within the range of previous clinical studies. A ten-miRNA signature (miR-31, miR-183, miR-184, miR-200c, miR-205, miR-210, miR-379, miR-424, miR-452, and miR-565) can separate CTC-positive samples of MBC cases from healthy control samples after epithelial cell enrichment. ${ }^{116}$ However, only miR-183 is detected in statistically significantly different amounts between CTC-positive and CTC-negative samples of MBC cases. ${ }^{116}$ Another study took a different approach and correlated changes in circulating miRNAs with the presence or absence of CTCs in MBC cases. Higher circulating amounts of seven miRNAs (miR-141, miR-200a, miR-200b, miR-200c, miR-203, miR-210, and miR-375) and lower amounts of miR768-3p were detected in plasma samples from CTC-positive MBC cases. ${ }^{117}$ miR-210 was the only overlapping miRNA between these two studies; epithelial cell-expressed miRNAs (miR-141, miR-200a, miR-200b, and miR-200c) did not reach statistical significance in the other study.

Changes in amounts of circulating miRNAs have been reported in various studies of blood samples collected before and after neoadjuvant treatment. Such changes could be useful in monitoring treatment response at an earlier time than current imaging technologies allow. However, there is no evidence at this time that circulating miRNA signatures would contain enough information to dissect molecular aberrations in individual metastatic lesions, which could be many and heterogeneous within the same patient. The amount of circulating miR-19a and miR-205 in serum before treatment correlated with response to neoadjuvant epirubicin + paclitaxel chemotherapy regimen in Stage II and III patients with luminal A breast tumors. ${ }^{118}$ Relatively lower levels of circulating miR-210 in plasma samples before treatment correlated with complete pathologic response to neoadjuvant trastuzumab treatment in patients with HER2 ${ }^{+}$breast tumors. ${ }^{119}$ At 24 weeks after surgery, the miR-210 in plasma samples of patients with residual disease (as assessed by pathological response) was reduced to the level of patients with complete pathological response. ${ }^{119}$ While circulating levels of miR-21, miR-29a, and miR-126 were relatively higher in plasma samples from breast cancer patients relative to those of healthy controls, there were no significant changes of these miRNAs between pre-surgery and post-surgery plasma samples. ${ }^{119}$ Another study found no correlation between the circulating amount of miR-21, miR-210, or miR-373 in serum samples before treatment and the response to neoadjuvant trastuzumab (or lapatinib) treatment in patients with HER2 ${ }^{+}$ breast tumors. ${ }^{120}$ In this study, however, relatively higher levels of circulating miR-21 in pre-surgery or post-surgery serum samples correlated with shorter overall survival. ${ }^{120}$ More studies are needed that carefully address the technical and biological reproducibility, as we discussed above for miRNA-based early-disease detection assays.

\section{Conclusion}

Breast cancer has been widely studied and characterized at the molecular level. Various molecular tools have already been incorporated into the clinic for diagnostic and prognostic applications based on gene (mRNA) and protein expression, but there are still unmet clinical needs for novel biomarkers that can improve diagnosis, management, and treatment. In this review, we provided a general look at the state of miRNA research on breast cancer. We limited our discussion to studies that associated miRNA changes with one of these focused challenges: early disease detection (Tables 1 and 2), management of a specific breast cancer subtype (Tables 3-5), or new opportunities to monitor and characterize MBC (Table 6). There are more studies that have linked altered expression of specific miRNAs with clinical outcome, but we did not review those that did not analyze their findings within the context of specific subtypes based on ER/PR/HER2 status.

The promise of miRNA biomarkers generates great enthusiasm. Their chemical stability in tissues, blood, and other body fluids, as well as their regulatory capacity to modulate target networks, are technically and biologically appealing. miRNA-based diagnostics have already reached the clinic in laboratory-developed tests that use qRT-PCR-based detection of miRNAs for differential diagnosis of pancreatic cancer, subtyping of lung and kidney cancers, and identification of the cell of origin for cancers having an unknown primary. ${ }^{121,122}$ For breast cancer applications, there is little agreement on the reported individual miRNAs and miRNA signatures among studies from either tissues or blood samples. We considered in detail parameters that may contribute to these discrepancies in blood samples. Most of these concerns also apply to tissue studies, namely, patient characteristics, experimental design, sample size, methodology, and analysis tools. Another limitation of most expression-profiling studies in whole-tissue 
specimens is that they measure collective levels of RNA from a mixture of different cell types. Intratumoral and intertumoral heterogeneity at the cellular and molecular levels are confounding factors in interpreting altered miRNA expression. This may explain in part the low overlap of reported miRNA signatures in tissues. We discussed the influence of altered miRNA expression in the stroma in the context of TNBC. Stromal features are known to influence cancer cell characteristics. ${ }^{123,124}$ Therefore, it is likely that miRNA-mediated regulation in other cellular compartments of the tumor microenvironment also influences cancer cells. Detection methods that incorporate the context of altered expression, such as multiplex ISH/immunohistochemistry assays, may provide additional validation tools for altered miRNA expression. ${ }^{13,93}$

In conclusion, it is premature to make specific recommendations for clinical implementation of miRNA biomarkers in managing breast cancer. More research is needed that includes multi-institutional participation and longitudinal studies of large patient cohorts, with well-annotated pathologic and clinical characteristics to validate the clinical value of miRNAs in breast cancer.

\section{Acknowledgment}

We thank David Nadziejka for technical editing.

\section{Disclosure}

The authors report no conflicts of interest in this work.

\section{References}

1. Prat A, Perou CM. Deconstructing the molecular portraits of breast cancer. Mol Oncol. 2011;5(1):5-23.

2. Tofigh A, Suderman M, Paquet ER, et al. The prognostic ease and difficulty of invasive breast carcinoma. Cell Rep. 2014;9(1):129-142.

3. Foulkes WD, Smith IE, Reis-Filho JS. Triple-negative breast cancer. N Engl J Med. 2010;363(20):1938-1948.

4. Dent R, Trudeau M, Pritchard KI, et al. Triple-negative breast cancer: clinical features and patterns of recurrence. Clin Cancer Res. 2007; 13(15 Pt 1):4429-4434.

5. Di Leva G, Garofalo M, Croce CM. MicroRNAs in cancer. Annu Rev Pathol. 2014;9:287-314.

6. Hausser J, Zavolan M. Identification and consequences of miRNA-target interactions - beyond repression of gene expression. Nat Rev Genet. 2014;15(9):599-612.

7. Machiela EP, Sempere LF. Individual noncoding RNA variations: their role in shaping and maintaining the epigenetic landscape. In: Tollefsbol T, editor. Personalized Epigenetics. Elsevier; in press.

8. Kita Y, Vincent K, Natsugoe S, Berindan-Neagoe I, Calin GA. Epigenetically regulated microRNAs and their prospect in cancer diagnosis. Expert Rev Mol Diagn. 2014;14(6):673-683.

9. Sempere LF, Freemantle S, Pitha-Rowe I, Moss E, Dmitrovsky E, Ambros V. Expression profiling of mammalian microRNAs uncovers a subset of brain-expressed microRNAs with possible roles in murine and human neuronal differentiation. Genome Biol. 2004;5(3):R13.

10. Tran N, Hutvagner G. Biogenesis and the regulation of the maturation of miRNAs. Essays Biochem. 2013;54:17-28.
11. Kozomara A, Griffiths-Jones S. miRBase: annotating high confidence microRNAs using deep sequencing data. Nucleic Acids Res. 2014; 42(Database issue):D68-D73.

12. De Cecco L, Dugo M, Canevari S, Daidone MG, Callari M. Measuring microRNA expression levels in oncology: from samples to data analysis. Crit Rev Oncog. 2013;18(4):273-287.

13. Zhang X, Lu X, Lopez-Berestein G, Sood A, Calin G. In situ hybridization-based detection of microRNAs in human diseases. microRNA Diagn Ther. 2013;1(1):12-23.

14. de Planell-Saguer M, Rodicio MC. Detection methods for microRNAs in clinic practice. Clin Biochem. 2013;46(10-11):869-878.

15. Pritchard CC, Cheng HH, Tewari M. MicroRNA profiling: approaches and considerations. Nat Rev Genet. 2012;13(5):358-369.

16. Howlader NN, Krapcho M, Garshell J, et al, editors. SEER Cancer Statistics Review, 1975-2011. National Cancer Institute; 2014. Available from: http://seer.cancer.gov/csr/1975_2011/. Accessed October 31, 2014.

17. Kilburn-Toppin F, Barter SJ. New horizons in breast imaging. Clin Oncol (R Coll Radiol). 2013;25(2):93-100.

18. Kerlikowske K, Zhu W, Hubbard RA, et al; Breast Cancer Surveillance Consortium. Outcomes of screening mammography by frequency, breast density, and postmenopausal hormone therapy. JAMA Intern Med. 2013;173(9):807-816.

19. Boyd NF, Guo H, Martin LJ, et al. Mammographic density and the risk and detection of breast cancer. $N$ Engl J Med. 2007;356(3): 227-236.

20. De Abreu FB, Wells WA, Tsongalis GJ. The emerging role of the molecular diagnostics laboratory in breast cancer personalized medicine. Am J Pathol. 2013;183(4):1075-1083.

21. Taylor DD, Gercel-Taylor C. The origin, function, and diagnostic potential of RNA within extracellular vesicles present in human biological fluids. Front Genet. 2013;4:142.

22. Haizhong M, Liang C, Wang G, et al. MicroRNA-mediated cancer metastasis regulation via heterotypic signals in the microenvironment. Curr Pharm Biotechnol. 2014;15(5):455-458.

23. Jarry J, Schadendorf D, Greenwood C, Spatz A, van Kempen LC. The validity of circulating microRNAs in oncology: five years of challenges and contradictions. Mol Oncol. 2014;8(4):819-829.

24. Dobbin KK. Statistical design and evaluation of biomarker studies. Methods Mol Biol. 2014;1102:667-677.

25. Wang K, Yuan Y, Cho JH, McClarty S, Baxter D, Galas DJ. Comparing the MicroRNA spectrum between serum and plasma. PLoS One. 2012;7(7):e41561.

26. Leidner RS, Li L, Thompson CL. Dampening enthusiasm for circulating microRNA in breast cancer. PLoS One. 2013;8(3):e57841.

27. Shen J, Hu Q, Schrauder M, et al. Circulating miR-148b and miR-133a as biomarkers for breast cancer detection. Oncotarget. 2014;5(14): 5284-5294.

28. Kodahl AR, Zeuthen P, Binder H, Knoop AS, Ditzel HJ. Alterations in circulating miRNA levels following early-stage estrogen receptorpositive breast cancer resection in post-menopausal women. PLoS One. 2014;9(7):e101950.

29. Sochor M, Basova P, Pesta M, et al. Oncogenic microRNAs: miR-155, miR-19a, miR-181b, and miR-24 enable monitoring of early breast cancer in serum. BMC Cancer. 2014;14:448.

30. Bruno AE, Li L, Kalabus JL, Pan Y, Yu A, Hu Z. miRdSNP: a database of disease-associated SNPs and microRNA target sites on 3'UTRs of human genes. BMC Genomics. 2012;13:44.

31. Ma XP, Zhang T, Peng B, Yu L, Jiang de K. Association between microRNA polymorphisms and cancer risk based on the findings of 66 case-control studies. PLoS One. 2013;8(11):e79584.

32. Xu Y, Gu L, Pan Y, et al. Different effects of three polymorphisms in MicroRNAs on cancer risk in Asian population: evidence from published literatures. PLoS One. 2013;8(6):e65123.

33. Yao S, Graham K, Shen J, et al. Genetic variants in microRNAs and breast cancer risk in African American and European American women. Breast Cancer Res Treat. 2013;141(3):447-459. 
34. Yang R, Schlehe B, Hemminki K, et al. A genetic variant in the premiR-27a oncogene is associated with a reduced familial breast cancer risk. Breast Cancer Res Treat. 2010;121(3):693-702.

35. Zhang N, Huo Q, Wang X, et al. A genetic variant in pre-miR-27a is associated with a reduced breast cancer risk in younger Chinese population. Gene. 2013;529(1):125-130.

36. Hu Z, Liang J, Wang Z, et al. Common genetic variants in premicroRNAs were associated with increased risk of breast cancer in Chinese women. Hum Mutat. 2009;30(1):79-84.

37. Catucci I, Yang R, Verderio P, et al. Evaluation of SNPs in miR-146a, miR196a2 and miR-499 as low-penetrance alleles in German and Italian familial breast cancer cases. Hum Mutat. 2010;31(1):E1052-E1057.

38. Chen J, Qin Z, Jiang Y, et al. Genetic variations in the flanking regions of miR-101-2 are associated with increased risk of breast cancer. PLoS One. 2014;9(1):e86319.

39. Paranjape T, Heneghan H, Lindner R, et al. A 3'-untranslated region KRAS variant and triple-negative breast cancer: a case-control and genetic analysis. Lancet Oncol. 2011;12(4):377-386.

40. Hollestelle A, Pelletier C, Hooning M, et al. Prevalence of the variant allele rs61764370 T $>\mathrm{G}$ in the 3'UTR of KRAS among Dutch BRCA1, BRCA2 and non-BRCA1/BRCA2 breast cancer families. Breast Cancer Res Treat. 2011;128(1):79-84.

41. Cerne JZ, Stegel V, Gersak K, Novakovic S. KRAS rs61764370 is associated with HER2-overexpressed and poorly-differentiated breast cancer in hormone replacement therapy users: a case control study. BMC Cancer. 2012;12:105.

42. Jiang Y, Qin Z, Hu Z, et al. Genetic variation in a hsa-let-7 binding site in RAD52 is associated with breast cancer susceptibility. Carcinogenesis. 2013;34(3):689-693.

43. Saetrom P, Biesinger J, Li SM, et al. A risk variant in an miR-125b binding site in BMPR1B is associated with breast cancer pathogenesis. Cancer Res. 2009;69(18):7459-7465.

44. Baumgarten SC, Frasor J. Minireview: Inflammation: an instigator of more aggressive estrogen receptor (ER) positive breast cancers. $\mathrm{Mol}$ Endocrinol. 2012;26(3):360-371.

45. Wolff AC, Hammond ME, Schwartz JN, et al; American Society of Clinical Oncology; College of American Pathologists. American Society of Clinical Oncology/College of American Pathologists guideline recommendations for human epidermal growth factor receptor 2 testing in breast cancer. J Clin Oncol. 2007;25(1):118-145.

46. Paik S, Kim C, Wolmark N. HER2 status and benefit from adjuvant trastuzumab in breast cancer. N Engl J Med. 2008;358(13):1409-1411.

47. Vogel CL, Cobleigh MA, Tripathy D, et al. Efficacy and safety of trastuzumab as a single agent in first-line treatment of HER2overexpressing metastatic breast cancer. J Clin Oncol. 2002;20(3): 719-726.

48. Slamon DJ, Leyland-Jones B, Shak S, et al. Use of chemotherapy plus a monoclonal antibody against HER2 for metastatic breast cancer that overexpresses HER2. N Engl J Med. 2001;344(11):783-792.

49. Cobleigh MA, Vogel CL, Tripathy D, et al. Multinational study of the efficacy and safety of humanized anti-HER2 monoclonal antibody in women who have HER2-overexpressing metastatic breast cancer that has progressed after chemotherapy for metastatic disease. J Clin Oncol. 1999;17(9):2639-2648.

50. Zhou J, Teng R, Wang Q, et al. Endocrine resistance in breast cancer: current status and a perspective on the roles of miRNAs (Review). Oncol Lett. 2013;6(2):295-305.

51. Wang SE, Lin RJ. MicroRNA and HER2-overexpressing Cancer. Microrna. 2013;2(2):137-147.

52. Yoshimoto N, Toyama T, Takahashi S, et al. Distinct expressions of microRNAs that directly target estrogen receptor alpha in human breast cancer. Breast Cancer Res Treat. 2011;130(1):331-339.

53. Rao X, Di Leva G, Li M, et al. MicroRNA-221/222 confers breast cancer fulvestrant resistance by regulating multiple signaling pathways. Oncogene. 2011;30(9):1082-1097.
54. Gan R, Yang Y, Yang X, Zhao L, Lu J, Meng QH. Downregulation of miR-221/222 enhances sensitivity of breast cancer cells to tamoxifen through upregulation of TIMP3. Cancer Gene Ther. 2014;21(7): 290-296.

55. Xiong J, Yu D, Wei N, et al. An estrogen receptor alpha suppressor, microRNA-22, is downregulated in estrogen receptor alpha-positive human breast cancer cell lines and clinical samples. FEBS J. 2010; 277(7):1684-1694

56. Lowery AJ, Miller N, Devaney A, et al. MicroRNA signatures predict oestrogen receptor, progesterone receptor and HER2/neu receptor status in breast cancer. Breast Cancer Res. 2009;11(3):R27.

57. Cittelly DM, Das PM, Spoelstra NS, et al. Downregulation of miR-342 is associated with tamoxifen resistant breast tumors. Mol Cancer. 2010;9:317.

58. Di Leva G, Piovan C, Gasparini P, et al. Estrogen mediated-activation of miR-191/425 cluster modulates tumorigenicity of breast cancer cells depending on estrogen receptor status. PLoS Genet. 2013;9(3): e1003311.

59. He YJ, Wu JZ, Ji MH, et al. miR-342 is associated with estrogen receptor-alpha expression and response to tamoxifen in breast cancer. Exp Ther Med. 2013;5(3):813-818.

60. Hoppe R, Achinger-Kawecka J, Winter S, et al. Increased expression of miR-126 and miR-10a predict prolonged relapse-free time of primary oestrogen receptor-positive breast cancer following tamoxifen treatment. Eur J Cancer. 2013;49(17):3598-3608.

61. Rodríguez-González FG, Sieuwerts AM, Smid M, et al. MicroRNA-30c expression level is an independent predictor of clinical benefit of endocrine therapy in advanced estrogen receptor positive breast cancer. Breast Cancer Res Treat. 2011;127(1):43-51.

62. Rothe F, Ignatiadis M, Chaboteaux C, et al. Global microRNA expression profiling identifies MiR-210 associated with tumor proliferation, invasion and poor clinical outcome in breast cancer. PLoS One. 2011;6(6):e20980.

63. Ward A, Shukla K, Balwierz A, et al. MicroRNA-519a is a novel oncomir conferring tamoxifen resistance by targeting a network of tumour-suppressor genes in ER+ breast cancer. J Pathol. 2014;233(4): 368-379.

64. Lyng MB, Laenkholm AV, Sokilde R, Gravgaard KH, Litman T, Ditzel HJ. Global microRNA expression profiling of high-risk ER+ breast cancers from patients receiving adjuvant tamoxifen mono-therapy: a DBCG study. PLoS One. 2012;7(5):e36170.

65. Foekens JA, Sieuwerts AM, Smid M, et al. Four miRNAs associated with aggressiveness of lymph node-negative, estrogen receptor-positive human breast cancer. Proc Natl Acad Sci U S A. 2008;105(35):13021-13026.

66. Toyama T, Kondo N, Endo Y, et al. High expression of microRNA-210 is an independent factor indicating a poor prognosis in Japanese triplenegative breast cancer patients. Jpn J Clin Oncol. 2012;42(4):256-263.

67. Buffa FM, Camps C, Winchester L, et al. microRNA associated progression pathways and potential therapeutic targets identified by integrated mRNA and microRNA expression profiling in breast cancer. Cancer Res. 2011;71(17):5635-5645.

68. Hong L, Yang J, Han Y, Lu Q, Cao J, Syed L. High expression of miR-210 predicts poor survival in patients with breast cancer: a metaanalysis. Gene. 2012;507(2):135-138.

69. Camps C, Buffa FM, Colella S, et al. hsa-miR-210 Is induced by hypoxia and is an independent prognostic factor in breast cancer. Clin Cancer Res. 2008;14(5):1340-1348.

70. Camps C, Saini HK, Mole DR, et al. Integrated analysis of microRNA and mRNA expression and association with HIF binding reveals the complexity of microRNA expression regulation under hypoxia. Mol Cancer. 2014;13:28.

71. Scott GK, Goga A, Bhaumik D, Berger CE, Sullivan CS, Benz CC. Coordinate suppression of ERBB2 and ERBB3 by enforced expression of micro-RNA miR-125a or miR-125b. J Biol Chem. 2007;282(2): $1479-1486$. 
72. Leivonen SK, Sahlberg KK, Mäkelä R, et al. High-throughput screens identify microRNAs essential for HER2 positive breast cancer cell growth. Mol Oncol. 2014;8(1):93-104.

73. Fang C, Zhao Y, Guo B. MiR-199b-5p targets HER2 in breast cancer cells. J Cell Biochem. 2013;114(7):1457-1463.

74. Iorio MV, Casalini P, Piovan C, et al. microRNA-205 regulates HER3 in human breast cancer. Cancer Res. 2009;69(6):2195-2200.

75. Ichikawa T, Sato F, Terasawa K, et al. Trastuzumab produces therapeutic actions by upregulating miR-26a and miR-30b in breast cancer cells PLoS One. 2012;7(2):e31422.

76. Le XF, Almeida MI, Mao W, et al. Modulation of MicroRNA-194 and cell migration by HER2-targeting trastuzumab in breast cancer. PLoS One. 2012;7(7):e41170.

77. Lee JA, Lee HY, Lee ES, Kim I, Bae JW. Prognostic Implications of MicroRNA-21 Overexpression in Invasive Ductal Carcinomas of the Breast. J Breast Cancer. 2011;14(4):269-275.

78. Persson H, Kvist A, Rego N, et al. Identification of new microRNAs in paired normal and tumor breast tissue suggests a dual role for the ERBB2/Her2 gene. Cancer Res. 2011;71(1):78-86.

79. Gong C, Yao Y, Wang Y, et al. Up-regulation of miR-21 mediates resistance to trastuzumab therapy for breast cancer. J Biol Chem. 2011;286(21):19127-19137.

80. Ye X, Bai W, Zhu H, et al. MiR-221 promotes trastuzumab-resistance and metastasis in HER2-positive breast cancers by targeting PTEN. BMB Rep. 2014;47(5):268-273.

81. Falkenberg N, Anastasov N, Rappl K, et al. MiR-221/-222 differentiate prognostic groups in advanced breast cancers and influence cell invasion. Br J Cancer. 2013;109(10):2714-2723.

82. Mattie MD, Benz CC, Bowers J, et al. Optimized high-throughput microRNA expression profiling provides novel biomarker assessment of clinical prostate and breast cancer biopsies. Mol Cancer. 2006;5:24

83. Gao LB, Bai P, Pan XM, et al. The association between two polymorphisms in pre-miRNAs and breast cancer risk: a meta-analysis. Breast Cancer Res Treat. 2011;125(2):571-574.

84. Gasparini P, Cascione L, Fassan M, et al. microRNA expression profiling identifies a four microRNA signature as a novel diagnostic and prognostic biomarker in triple negative breast cancers. Oncotarget. 2014;5(5):1174-1184.

85. Kolacinska A, Morawiec J, Pawlowska Z, et al. Association of microRNA-93, 190, 200b and receptor status in core biopsies from stage III breast cancer patients. DNA Cell Biol. 2014;33(9):624-629.

86. Ouyang M, Li Y, Ye S, et al. MicroRNA profiling implies new markers of chemoresistance of triple-negative breast cancer. PLoS One. 2014; 9(5):e96228.

87. Sempere LF, Christensen M, Silahtaroglu A, et al. Altered MicroRNA expression confined to specific epithelial cell subpopulations in breast cancer. Cancer Res. 2007;67(24):11612-11620.

88. Sempere LF, Preis M, Yezefski T, et al. Fluorescence-based codetection with protein markers reveals distinct cellular compartments for altered MicroRNA expression in solid tumors. Clin Cancer Res. 2010;16(16):4246-4255.

89. Preis M, Gardner TB, Gordon SR, et al. microRNA-10b expression correlates with response to neoadjuvant therapy and survival in pancreatic ductal adenocarcinoma. Clin Cancer Res. 2011;17(17): 5812-5821.

90. Jørgensen S, Baker A, Moller S, Nielsen BS. Robust one-day in situ hybridization protocol for detection of microRNAs in paraffin samples using LNA probes. Methods. 2010;52(4):375-381.

91. Rask L, Balslev E, Jørgensen S, et al. High expression of miR-21 in tumor stroma correlates with increased cancer cell proliferation in human breast cancer. APMIS. 2011;119(10):663-673.

92. Susman S, Tomuleasa C, Fekete Z, Pilato B, Irimie A, Berindan-Neagoe I. The importance of microRNAs in the stroma-breast cancer cell interplay. Cancer Biomark. 2014;14(2-3):137-144.
93. Sempere LF. Tissue slide-based microRNA characterization of tumors: how detailed could diagnosis become for cancer medicine? Expert Rev Mol Diagn. 2014;14(7):853-869.

94. Gong C, Nie Y, Qu S, et al. miR-21 induces myofibroblast differentiation and promotes the malignant progression of breast phyllodes tumors. Cancer Res. 2014;74(16):4341-4352.

95. Bullock MD, Pickard KM, Nielsen BS, et al. Pleiotropic actions of miR-21 highlight the critical role of deregulated stromal microRNAs during colorectal cancer progression. Cell Death Dis. 2013;4:e684.

96. Kadera BE, Li L, Toste PA, et al. MicroRNA-21 in pancreatic ductal adenocarcinoma tumor-associated fibroblasts promotes metastasis. PLoS One. 2013;8(8):e71978.

97. Mackenzie TA, Schwartz G, Calderone HM, et al. Stromal expression of miR-21 identifies high-risk group in triple-negative breast cancer Am J Pathol. 2014;184(12):3217-3225.

98. Wang L, Wang J. MicroRNA-mediated breast cancer metastasis: from primary site to distant organs. Oncogene. 2012;31(20):2499-2511.

99. Ma L, Reinhardt F, Pan E, et al. Therapeutic silencing of miR-10b inhibits metastasis in a mouse mammary tumor model. Nat Biotechnol. 2010;28(4):341-347.

100. Ma L, Teruya-Feldstein J, Weinberg RA. Tumour invasion and metastasis initiated by microRNA-10b in breast cancer. Nature. 2007;449(7163):682-688.

101. Ahmad A, Sethi S, Chen W, Ali-Fehmi R, Mittal S, Sarkar FH. Up-regulation of microRNA-10b is associated with the development of breast cancer brain metastasis. Am J Transl Res. 2014;6(4): 384-390.

102. Parrella P, Barbano R, Pasculli B, et al. Evaluation of microRNA-10b prognostic significance in a prospective cohort of breast cancer patients. Mol Cancer. 2014;13:142.

103. Chen W, Cai F, Zhang B, Barekati Z, Zhong XY. The level of circulating miRNA-10b and miRNA-373 in detecting lymph node metastasis of breast cancer: potential biomarkers. Tumour Biol. 2013;34(1):455-462

104. Zhao FL, Hu GD, Wang XF, Zhang XH, Zhang YK, Yu ZS. Serum overexpression of microRNA-10b in patients with bone metastatic primary breast cancer. $J$ Int Med Res. 2012;40(3):859-866.

105. Anfossi S, Giordano A, Gao H, et al. High serum miR-19a levels are associated with inflammatory breast cancer and are predictive of favorable clinical outcome in patients with metastatic HER2+ inflammatory breast cancer. PLoS One. 2014;9(1):e83113.

106. Markou A, Yousef GM, Stathopoulos E, Georgoulias V, Lianidou E. Prognostic significance of metastasis-related microRNAs in early breast cancer patients with a long follow-up. Clin Chem. 2014;60(1): 197-205.

107. Gee HE, Camps C, Buffa FM, et al. MicroRNA-10b and breast cancer metastasis. Nature. 2008;455(7216):E8-E9; author reply E9.

108. Gravgaard KH, Lyng MB, Laenkholm AV, et al. The miRNA-200 family and miRNA-9 exhibit differential expression in primary versus corresponding metastatic tissue in breast cancer. Breast Cancer Res Treat. 2012;134(1):207-217.

109. Ryu S, McDonnell K, Choi H, et al. Suppression of miRNA-708 by polycomb group promotes metastases by calcium-induced cell migration. Cancer Cell. 2013;23(1):63-76.

110. Okuda H, Xing F, Pandey PR, et al. miR-7 suppresses brain metastasis of breast cancer stem-like cells by modulating KLF4. Cancer Res. 2013;73(4):1434-1444.

111. Krutilina R, Sun W, Sethuraman A, et al. MicroRNA-18a inhibits hypoxia-inducible factor 1-alpha activity and lung metastasis in basal breast cancers. Breast Cancer Res. 2014;16(4):R78.

112. Chou J, Lin JH, Brenot A, Kim JW, Provot S, Werb Z. GATA3 suppresses metastasis and modulates the tumour microenvironment by regulating microRNA-29b expression. Nat Cell Biol. 2013;15(2):201-213.

113. Pellegrino L, Stebbing J, Braga VM, et al. miR-23b regulates cytoskeletal remodeling, motility and metastasis by directly targeting multiple transcripts. Nucleic Acids Res. 2013;41(10):5400-5412. 
114. Ell B, Qiu Q, WeiY, et al. The MicroRNA-23b/27b/24 cluster promotes breast cancer lung metastasis by targeting metastasis-suppressive gene prosaposin. J Biol Chem. 2014;289(32):21888-21895.

115. Mostert B, Sieuwerts AM, Martens JW, Sleijfer S. Diagnostic applications of cell-free and circulating tumor cell-associated miRNAs in cancer patients. Expert Rev Mol Diagn. 2011;11(3):259-275.

116. Sieuwerts AM, Mostert B, Bolt-de Vries J, et al. mRNA and microRNA expression profiles in circulating tumor cells and primary tumors of metastatic breast cancer patients. Clin Cancer Res. 2011; 17(11):3600-3618.

117. Madhavan D, Zucknick M, Wallwiener M, et al. Circulating miRNAs as surrogate markers for circulating tumor cells and prognostic markers in metastatic breast cancer. Clin Cancer Res. 2012;18(21):5972-5982.

118. Li Q, Liu M, Ma F, et al. Circulating miR-19a and miR-205 in serum may predict the sensitivity of luminal A subtype of breast cancer patients to neoadjuvant chemotherapy with epirubicin plus paclitaxel. PLoS One. 2014;9(8):e104870.

119. Jung EJ, Santarpia L, Kim J, et al. Plasma microRNA 210 levels correlate with sensitivity to trastuzumab and tumor presence in breast cancer patients. Cancer. 2012;118(10):2603-2614.

120. Muller V, Gade S, Steinbach B, et al. Changes in serum levels of miR-21, miR-210, and miR-373 in HER2-positive breast cancer patients undergoing neoadjuvant therapy: a translational research project within the Geparquinto trial. Breast Cancer Res Treat. 2014;147(1): $61-68$.

121. Asuragen [webpage on the Internet]. miRInform ${ }^{\circledR}$ Pancreas. Austin, TX: Asuragen, Inc.; 2014. Available from: http://asuragen.com/ products-and-services/clinical-lab/mirinform-pancreas/. Accessed October 31, 2014.

122. Rosetta Genomics [webpage on the Internet]. MicroRNAs in Diagnostics. Philadelphia, PA: Rosetta Genomics Inc. Available from: http://www.rosettagenomics.com/micrornas-in-diagnostics. Accessed October 31, 2014.

123. Finak G, Bertos N, Pepin F, et al. Stromal gene expression predicts clinical outcome in breast cancer. Nat Med. 2008; 14(5):518-527.

124. Kristensen VN, Vaske CJ, Ursini-Siegel J, et al. Integrated molecular profiles of invasive breast tumors and ductal carcinoma in situ (DCIS) reveal differential vascular and interleukin signaling. Proc Natl Acad Sci U S A. 2012;109(8):2802-2807.

125. Joosse SA, Muller V, Steinbach B, Pantel K, Schwarzenbach H. Circulating cell-free cancer-testis MAGE-A RNA, BORIS RNA, let-7b and miR-202 in the blood of patients with breast cancer and benign breast diseases. Br J Cancer. 2014;111(5):909-917.

126. Chan M, Liaw CS, Ji SM, et al. Identification of circulating microRNA signatures for breast cancer detection. Clin Cancer Res. 2013;19(16):4477-4487.

127. Mar-Aguilar F, Mendoza-Ramirez JA, Malagón-Santiago I, et al. Serum circulating microRNA profiling for identification of potential breast cancer biomarkers. Dis Markers. 2013;34(3):163-169.

128. Kodahl AR, Lyng MB, Binder $\mathrm{H}$, et al. Novel circulating microRNA signature as a potential non-invasive multi-marker test in ER-positive early-stage breast cancer: a case control study. Mol Oncol. 2014;8(5): 874-883.

129. Guo L, Zhao Y, Yang S, Cai M, Wu Q, Chen F. Genome-wide screen for aberrantly expressed miRNAs reveals miRNA profile signature in breast cancer. Mol Biol Rep. 2013;40(3):2175-2186.

130. Godfrey AC, Xu Z, Weinberg CR, et al. Serum microRNA expression as an early marker for breast cancer risk in prospectively collected samples from the Sister Study cohort. Breast Cancer Res. 2013;15(3):R42.

131. Gao J, Zhang Q, Xu J, Guo L, Li X. Clinical significance of serum miR-21 in breast cancer compared with CA153 and CEA. Chin $J$ Cancer Res. 2013;25(6):743-748.

132. Si H, Sun X, Chen Y, et al. Circulating microRNA-92a and microRNA-21 as novel minimally invasive biomarkers for primary breast cancer. J Cancer Res Clin Oncol. 2013;139(2):223-229.
133. Luo J, Zhao Q, Zhang W, et al. A novel panel of microRNAs provides a sensitive and specific tool for the diagnosis of breast cancer. Mol Med Rep. 2014;10(2):785-791.

134. Zeng RC, Zhang W, Yan XQ, et al. Down-regulation of miRNA-30a in human plasma is a novel marker for breast cancer. Med Oncol. 2013;30(1):477.

135. Cuk K, Zucknick M, Madhavan D, et al. Plasma microRNA panel for minimally invasive detection of breast cancer. PLoS One. 2013;8(10):e76729.

136. Waters PS, Dwyer RM, Brougham C, et al. Impact of tumour epithelial subtype on circulating microRNAs in breast cancer patients. PLoS One. 2014;9(3):e90605.

137. Cuk K, Zucknick M, Heil J, et al. Circulating microRNAs in plasma as early detection markers for breast cancer. Int $J$ Cancer. 2013;132(7):1602-1612.

138. Liu J, Mao Q, Liu Y, Hao X, Zhang S, Zhang J. Analysis of miR-205 and miR-155 expression in the blood of breast cancer patients. Chin $J$ Cancer Res. 2013;25(1):46-54.

139. Wang PY, Gong HT, Li BF, et al. Higher expression of circulating miR-182 as a novel biomarker for breast cancer. Oncol Lett. 2013;6(6): 1681-1686.

140. Zearo S, Kim E, Zhu Y, et al. MicroRNA-484 is more highly expressed in serum of early breast cancer patients compared to healthy volunteers. BMC Cancer. 2014;14:200.

141. Chen QH, Wang QB, Zhang B. Ethnicity modifies the association between functional microRNA polymorphisms and breast cancer risk: a HuGE meta-analysis. Tumour Biol. 2014;35(1):529-543.

142. Kontorovich T, Levy A, Korostishevsky M, Nir U, Friedman E. Single nucleotide polymorphisms in miRNA binding sites and miRNA genes as breast/ovarian cancer risk modifiers in Jewish high-risk women. Int J Cancer. 2010;127(3):589-597.

143. Zhong S, Chen Z, Xu J, Li W, Zhao J. Pre-mir-27a rs895819 polymorphism and cancer risk: a meta-analysis. Mol Biol Rep. 2013;40(4): 3181-3186.

144. Xu Q, He CY, Liu JW, Yuan Y. Pre-miR-27a rs895819A/G polymorphisms in cancer: a meta-analysis. PLoS One. 2013;8(6): e65208.

145. Bensen JT, Tse CK, Nyante SJ, Barnholtz-Sloan JS, Cole SR, Millikan RC. Association of germline microRNA SNPs in premiRNA flanking region and breast cancer risk and survival: the Carolina Breast Cancer Study. Cancer Causes Control. 2013;24(6): 1099-1109.

146. Pastrello C, Polesel J, Della Puppa L, Viel A, Maestro R. Association between hsa-mir-146a genotype and tumor age-of-onset in BRCA1/ BRCA2-negative familial breast and ovarian cancer patients. Carcinogenesis. 2010;31(12):2124-2126.

147. Wang PY, Gao ZH, Jiang ZH, Li XX, Jiang BF, Xie SY. The associations of single nucleotide polymorphisms in miR-146a, miR-196a and miR-499 with breast cancer susceptibility. PLoS One. 2013;8(9): e70656.

148. Lee SJ, Seo JW, Chae YS, et al. Genetic polymorphism of miR-196a as a prognostic biomarker for early breast cancer. Anticancer Res. 2014;34(6):2943-2949.

149. Linhares JJ, Azevedo M Jr, Siufi AA, et al. Evaluation of single nucleotide polymorphisms in microRNAs (hsa-miR-196a2 rs11614913 C/T) from Brazilian women with breast cancer. BMC Med Genet. 2012;13:119.

150. Smith RA, Jedlinski DJ, Gabrovska PN, Weinstein SR, Haupt L, Griffiths LR. A genetic variant located in miR-423 is associated with reduced breast cancer risk. Cancer Genomics Proteomics. 2012;9(3): $115-118$

151. Chen P, Zhang J, Zhou F. miR-499 rs3746444 polymorphism is associated with cancer development among Asians and related to breast cancer susceptibility. Mol Biol Rep. 2012;39(12):10433-10438.

152. Sun H, Li Q, Yang T, Wang W. Quantitative assessment of the association between microRNA-499 rs3746444 A/G polymorphism and cancer risk. Tumour Biol. 2014;35(3):2351-2358. 
153. Fan $\mathrm{C}$, Chen $\mathrm{C}, \mathrm{Wu} \mathrm{D}$. The association between common genetic variant of microRNA-499 and cancer susceptibility: a meta-analysis. Mol Biol Rep. 2013;40(4):3389-3394.

154. Nicoloso MS, Sun H, Spizzo R, et al. Single-nucleotide polymorphisms inside microRNA target sites influence tumor susceptibility. Cancer Res. 2010;70(7):2789-2798.

155. He N, Zheng H, Li P, et al. miR-485-5p binding site SNP rs8752 in HPGD gene is associated with breast cancer risk. PLoS One. 2014;9(7):e102093.

156. Gilam A, Edry L, Mamluk-Morag E, et al. Involvement of IGF-1R regulation by miR-515-5p modifies breast cancer risk among BRCA1 carriers. Breast Cancer Res Treat. 2013;138(3):753-760.

157. Wang L, Liu W, Jiang W, et al. A miRNA binding site singlenucleotide polymorphism in the $3^{\prime}$-UTR region of the IL23R gene is associated with breast cancer. PLoS One. 2012;7(12):e49823.

158. Zheng J, Jiang L, Zhang L, et al. Functional genetic variations in the IL-23 receptor gene are associated with risk of breast, lung and nasopharyngeal cancer in Chinese populations. Carcinogenesis. 2012;33(12):2409-2416.

159. Zheng H, Song F, Zhang L, et al. Genetic variants at the miR-124 binding site on the cytoskeleton-organizing IQGAP1 gene confer differential predisposition to breast cancer. Int J Oncol. 2011;38(4): 1153-1161.

160. Brendle A, Lei H, Brandt A, et al. Polymorphisms in predicted microRNA-binding sites in integrin genes and breast cancer: ITGB4 as prognostic marker. Carcinogenesis. 2008;29(7):1394-1399.

161. Zhang L, Liu Y, Song F, et al. Functional SNP in the microRNA-367 binding site in the $3^{\prime} \mathrm{UTR}$ of the calcium channel ryanodine receptor gene 3 (RYR3) affects breast cancer risk and calcification. Proc Natl Acad Sci U S A. 2011;108(33):13653-13658.

162. Zhang BL, Song FJ, Zheng H, Zhang LN, Zhao YR, Chen KX. [SNP rs16917496 within SET8 3'UTR is associated with the age of onset of breast cancer]. Zhonghua Zhong Liu Za Zhi. 2012;34(11):835-837. Chinese.
163. Quesne JL, Jones J, Warren J, et al. Biological and prognostic associations of miR-205 and let-7b in breast cancer revealed by in situ hybridization analysis of micro-RNA expression in arrays of archival tumour tissue. J Pathol. 2012;227(3):306-314.

164. Radojicic J, Zaravinos A, Vrekoussis T, Kafousi M, Spandidos DA, Stathopoulos EN. MicroRNA expression analysis in triple-negative (ER, PR and Her2/neu) breast cancer. Cell Cycle. 2011;10(3): 507-517.

165. Cascione L, Gasparini P, Lovat F, et al. Integrated microRNA and mRNA signatures associated with survival in triple negative breast cancer. PLoS One. 2013;8(2):e55910.

166. Dong G, Liang X, Wang D, et al. High expression of miR-21 in triple-negative breast cancers was correlated with a poor prognosis and promoted tumor cell in vitro proliferation. Med Oncol. 2014; 31(7):57.

167. Svoboda M, Sana J, Redova M, et al. MiR-34b is associated with clinical outcome in triple-negative breast cancer patients. Diagn Pathol. 2012;7:31.

168. Berber U, Yilmaz I, Narli G, Haholu A, Kucukodaci Z, Demirel D. miR-205 and miR-200c: predictive micro RNAs for lymph node metastasis in triple negative breast cancer. J Breast Cancer. 2014; 17(2):143-148

169. de Rinaldis E, Gazinska P, Mera A, et al. Integrated genomic analysis of triple-negative breast cancers reveals novel microRNAs associated with clinical and molecular phenotypes and sheds light on the pathways they control. BMC Genomics. 2013;14:643.

170. Eichelser C, Flesch-Janys D, Chang-Claude J, Pantel K, Schwarzenbach H. Deregulated serum concentrations of circulating cell-free microRNAs miR-17, miR-34a, miR-155, and miR-373 in human breast cancer development and progression. Clin Chem. 2013; 59(10):1489-1496.

171. Tang D, Zhang Q, Zhao S, et al. The expression and clinical significance of microRNA-1258 and heparanase in human breast cancer. Clin Biochem. 2013;46(10-11):926-932.
Breast Cancer: Targets and Therapy

\section{Publish your work in this journal}

Breast Cancer: Targets and Therapy is an international, peerreviewed open access journal focusing on breast cancer research, identification of therapeutic targets and the optimal use of preventative and integrated treatment interventions to achieve improved outcomes, enhanced survival and quality of life for the cancer patient.

\section{Dovepress}

View the full aims and scopes of this journal here. The manuscript management system is completely online and includes a very quick and fair peer-review system, which is all easy to use. Visit http:// www.dovepress.com/testimonials.php to read real quotes from published authors. 